\title{
THE DECOMPOSITION OF LIE POWERS
}

\author{
R. M. BRYANT and M. SCHOCKER
}

\begin{abstract}
Let $G$ be a group, $F$ a field of prime characteristic $p$ and $V$ a finite-dimensional $F G$ module. Let $L(V)$ denote the free Lie algebra on $V$ regarded as an $F G$-submodule of the free associative algebra (or tensor algebra) $T(V)$. For each positive integer $r$, let $L^{r}(V)$ and $T^{r}(V)$ be the $r$ th homogeneous components of $L(V)$ and $T(V)$, respectively. Here $L^{r}(V)$ is called the $r$ th Lie power of $V$. Our main result is that there are submodules $B_{1}, B_{2}, \ldots$ of $L(V)$ such that, for all $r, B_{r}$ is a direct summand of $T^{r}(V)$ and, whenever $m \geqslant 0$ and $k$ is not divisible by $p$,

$$
L^{p^{m} k}(V)=L^{p^{m}}\left(B_{k}\right) \oplus L^{p^{m-1}}\left(B_{p k}\right) \oplus \cdots \oplus L^{p}\left(B_{p^{m-1} k}\right) \oplus L^{1}\left(B_{p^{m} k}\right) .
$$

Thus every Lie power is a direct sum of Lie powers of $p$-power degree. The approach builds on an analysis of $T^{r}(V)$ as a bimodule for $G$ and the Solomon descent algebra.
\end{abstract}

\section{Introduction}

Let $G$ be a group and $F$ a field. For any finite-dimensional $F G$-module $V$ let $L(V)$ be the free Lie algebra on $V$. Then $L(V)$ may be regarded as an $F G$-module on which each element of $G$ acts as a Lie algebra automorphism. Furthermore, each homogeneous component $L^{r}(V)$ is a finite-dimensional submodule, called the $r$ th Lie power of $V$. We regard $L(V)$ as an $F G$-submodule of the free associative algebra, or tensor algebra, $T(V)$. Thus $L^{r}(V)$ is a submodule of $T^{r}(V)$.

In characteristic 0 , the structure of $L^{r}(V)$ has been clarified by a number of papers, including those of Brandt [3], Klyachko [19], and Kraskiewicz and Weyman [20]. We concentrate here on the more difficult case where $F$ has prime characteristic $p$. When $r$ is not divisible by $p, L^{r}(V)$ is comparatively well understood. In

Research of second author supported by Deutsche Forschungsgemeinschaft (DFG Scho-799). 2000 Mathematics Subject Classification. Primary 17B01, Secondary 20C07, 20 C20. 
this case, $L^{r}(V)$ is a direct summand of $T^{r}(V)$ (that is, $L^{r}(V)$ has a complement in $\left.T^{r}(V)\right)$ and it is possible to exploit knowledge of $T^{r}(V)$. For example, when $F$ is infinite and $G$ is the general linear group on $V$, the indecomposable summands of $L^{r}(V)$, for $r$ not divisible by $p$, were described up to isomorphism by Donkin and Erdmann [13].

The first case beyond this, namely the case $r=p$, was studied by Bryant and Stöhr [10], by means of a detailed analysis of $T^{p}(V)$. Further progress was made by Erdmann and Schocker [14] who studied the case $r=p k$ where $k$ is not divisible by $p$. Their methods make substantial use of Solomon's descent algebra, showing that its significance for free Lie algebras goes beyond the case of characteristic 0 . Their main results are concerned with the relationship between $L^{p k}(V)$ and $L^{k}(V)$. As a consequence, they were able to prove the factorisation $\Phi_{F G}^{p k}=\Phi_{F G}^{p} \circ \Phi_{F G}^{k}$ for the "Lie resolvents" introduced by Bryant [4]. Such factorisations, for certain groups $G$, first appeared in [5] and [6]. It follows from the results of [14] that the study of $L^{p k}(V)$ can be reduced, broadly speaking, to the study of $L^{p}\left(L^{k}(V)\right)$. As we have already seen, $L^{k}(V)$ is comparatively well understood. Thus the study of $p k$ th Lie powers is reduced, in a sense, to the study of $p$ th Lie powers.

This theme is developed a great deal further in the present paper. We show that the study of arbitrary Lie powers may be reduced, in the same sense, to the study of Lie powers of $p$-power degree.

First we develop some aspects of the Poincaré-Birkhoff-Witt Theorem. Linked with this theorem there is a filtration of $T^{r}(V)$ by $F G$-submodules in which the factors are isomorphic to certain tensor products of symmetric powers of Lie powers of $V$, called "higher Lie modules". At the bottom of the filtration is $L^{r}(V)$ and, at the top, the $r$ th symmetric power $S^{r}(V)$. Several papers on free Lie algebras ([10], for example) have used this filtration to study $L^{r}(V)$, exploiting the fact that $T^{r}(V)$ is comparatively well understood. The weakness of this method is that the filtration has many terms.

Our first result, called the "Filtration Theorem" and proved in $\S 3$, does something to remedy this weakness. We show that the filtration can be modified by means of primitive idempotents of the modular descent algebra. It turns out that $T^{r}(V)$ splits up as a direct sum of $F G$-submodules each with a much shorter 
filtration than before. The summands of $T^{r}(V)$ correspond to the primitive idempotents, and these are indexed by the "p-equivalence classes" of partitions of $r$.

In $\S 4$, we prove our main result about free Lie algebras, called the "Decomposition Theorem". This reduces the study of arbitrary Lie powers to the study of Lie powers of $p$-power degree and may be stated as follows. Let $F$ be a field of characteristic $p$. Let $G$ be a group and let $V$ be a finite-dimensional $F G$-module. Then, for each positive integer $r$, there is a submodule $B_{r}$ of $L^{r}(V)$ such that $B_{r}$ is a direct summand of $T^{r}(V)$ and, for $k$ not divisible by $p$ and $m \geqslant 0$,

$$
L^{p^{m} k}(V)=L^{p^{m}}\left(B_{k}\right) \oplus L^{p^{m-1}}\left(B_{p k}\right) \oplus \cdots \oplus L^{1}\left(B_{p^{m} k}\right)
$$

This has to be interpreted with a little care. As we shall see in $\S 2$, the subalgebra of $L(V)$ generated by $B_{r}$ is free on $B_{r}$. It may therefore be identified with $L\left(B_{r}\right)$ and then we have $L^{i}\left(B_{r}\right) \subseteq L^{i r}(V)$ for all $i$. The proof of the Decomposition Theorem uses the Filtration Theorem of $\S 3$ as well as Lazard's "Elimination Theorem" (see $\S 2$ below). This last result has proved to be fundamental in the theory and has been repeatedly used in earlier work such as [7].

In another paper $[\mathbf{9}]$ we shall obtain further information about the isomorphism types of the modules $B_{r}$ and we shall show that the Decomposition Theorem yields the factorisation of Lie resolvents $\Phi_{F G}^{p^{m} k}=\Phi_{F G}^{p^{m}} \circ \Phi_{F G}^{k}$ for every non-negative integer $m$ and every positive integer $k$ not divisible by $p$.

\section{Preliminaries}

We shall require some technical variations on known material concerned with free Lie algebras and representations of the general linear group. These are collected together in the present section.

We first work over a ring $R$ which is allowed to be either the ring of integers or an arbitrary field. (This is simply a sufficient degree of generality for our purposes.) Let $V$ be a free $R$-module. We write $T(V)$ for the free associative $R$-algebra (with identity element) freely generated by any $R$-basis of $V$. Thus $T(V)$ has an $R$ module decomposition $T(V)=\bigoplus_{r \geqslant 0} T^{r}(V)$ where, for each $r, T^{r}(V)$ is the $r$ th homogeneous component of $T(V)$ and we identify $T^{1}(V)$ with $V$. Each $T^{r}(V)$ is 
a free $R$-module and $T^{r}(V) \cong V \otimes_{R} \cdots \otimes_{R} V$, with $r$ factors. We call $T^{r}(V)$ the $r$ th tensor power of $V$. Note, however, that we write $a b$ rather than $a \otimes b$ for the product of elements $a$ and $b$ of $T(V)$. For $a \in T^{r}(V)$ we write $\operatorname{deg} a=r$.

The algebra $T(V)$ may be made into a Lie $R$-algebra by means of the bracket operation given by $[a, b]=a b-b a$. The Lie subalgebra of $T(V)$ generated by $V$ is denoted by $L(V)$. By a theorem of Witt (see [18] for the case of a field and [2] for general $R), L(V)$ is a free Lie $R$-algebra, freely generated by any basis of $V$, and $T(V)$ is the enveloping algebra of $L(V)$. For $r \geqslant 1$, we write $L^{r}(V)=T^{r}(V) \cap L(V)$. Thus $L(V)=\bigoplus_{r \geqslant 1} L^{r}(V)$, where $L^{1}(V)=V$. Each $L^{r}(V)$ is a free $R$-module, called the $r$ th Lie power of $V$.

Let $S(V)$ denote the free commutative associative $R$-algebra (that is, polynomial ring) freely generated by any $R$-basis of $V$. Then, as for $T(V)$, we have $S(V)=\bigoplus_{r \geqslant 0} S^{r}(V)$. Each $S^{r}(V)$ is a free $R$-module called the $r$ th symmetric power of $V$.

When $V$ has finite rank then $T^{r}(V), L^{r}(V)$ and $S^{r}(V)$ also have finite rank.

Suppose that $G$ is a group and that the free $R$-module $V$ is a (right) $R G$ module. The action of $G$ on $V$ extends to $T(V)$ so that $G$ acts by algebra automorphisms. Thus $T(V)$ becomes an $R G$-module. The subspaces $L(V), T^{r}(V)$ and $L^{r}(V)$ are $G$-invariant, so they also become $R G$-modules. Similarly, $S(V)$ and the subspaces $S^{r}(V)$ can be given the structure of $R G$-modules.

We now consider the case $R=F$, where $F$ is a field. Suppose that $B$ is a subspace of $T^{r}(V)$ for some $r \geqslant 1$. Then it is easily verified that the subalgebra of $T(V)$ generated by $B$ is isomorphic to $T(B)$ and may be identified with $T(B)$ by means of an isomorphism which is the identity on $B$. Then $T^{i}(B) \subseteq T^{i r}(V)$ for all $i \geqslant 0$. Furthermore, if $B \subseteq L^{r}(V)$ then the Lie subalgebra of $L(V)$ generated by $B$ is equal to the subalgebra $L(B)$ of $T(B)$ as previously defined. Thus it is a free Lie algebra. Furthermore, $L^{i}(B) \subseteq L^{i r}(V)$ for all $i \geqslant 1$. This is the justification for the notation used in the Decomposition Theorem as stated in $\S 1$.

If $L$ is a free Lie algebra over $F$ and $W$ is a subspace of $L$ such that some basis (equivalently, every basis) of $W$ is a free generating set of $L$ then we say (with slight abuse of language) that $L$ is free on $W$ or that $L$ is freely generated by $W$. In this case $L$ is isomorphic to $L(W)$. 
If $L$ is a free Lie algebra over $F$ and $Q$ is a subalgebra of $L$ then, by the theorem of Shirshov and Witt (see [21, Theorem 2.5]), $Q$ is free (on some free generating set). If $W$ is a subspace of $L$ then the subalgebra of $L$ generated by $W$ is not necessarily free on $W$ (though it is free on some subspace). If this subalgebra is free on $W$ we write it as $L(W)$. Indeed we identify the subalgebra generated by $W$ with $L(W)$ by means of the isomorphism which is the identity on $W$.

The following result is essentially well known and is given as [8, Lemma 1].

Lemma 2.1. Let $L$ be a free Lie algebra over $F$ and, for each $r \geqslant 1$, let $L_{r}$ be the rth homogeneous component of $L$ with respect to a given free generating set. Let $Q$ be a subalgebra of $L$ of the form $Q=Q_{1} \oplus Q_{2} \oplus \cdots$ with $Q_{r}=Q \cap L_{r}$ for all $r$. For each $r \geqslant 1$, let $Q(r)$ denote the subalgebra of $Q$ generated by $Q_{1} \oplus \cdots \oplus Q_{r}$, and write $Q(0)=0$. For each $r \geqslant 1$, let $W_{r}$ be any subspace of $Q_{r}$ satisfying $Q_{r}=\left(Q(r-1) \cap Q_{r}\right) \oplus W_{r}$. Then $Q$ is free on $W_{1} \oplus W_{2} \oplus \cdots$, that is,

$$
Q=L\left(W_{1} \oplus W_{2} \oplus \cdots\right) .
$$

For subspaces $B$ and $C$ of any Lie algebra over $F$, let $C \imath B$ denote the subspace defined by

$$
C \imath B=C+[C, B]+[C, B, B]+\cdots .
$$

(We use the left-normed convention for Lie products.) The following lemma is a version of Lazard's "Elimination Theorem" (see [2, Chapter 2, §2.9, Proposition $10]$ ). In the form written here it is a special case (with trivial group action) of [ 7 , Lemma 2.2] or [8, Lemma 2].

Lemma 2.2. Let $B$ and $C$ be $F$-spaces, and consider the free Lie algebra $L(B \oplus C)$. Then $B$ and $C \imath B$ freely generate subalgebras $L(B)$ and $L(C \imath B)$, and there is an F-space decomposition $L(B \oplus C)=L(B) \oplus L(C \curlywedge B)$. Furthermore,

$$
C \imath B=C \oplus[C, B] \oplus[C, B, B] \oplus \cdots
$$

and, for each $n \geqslant 0$, there is an F-space isomorphism

$$
\zeta_{n}:[C, \underbrace{B, \ldots, B}_{n}] \stackrel{\cong}{\cong} \otimes \underbrace{B \otimes \cdots \otimes B}_{n}
$$


such that $\zeta_{n}\left(\left[c, b_{1}, \ldots, b_{n}\right]\right)=c \otimes b_{1} \otimes \cdots \otimes b_{n}$ for all $c \in C$ and $b_{1}, \ldots, b_{n} \in B$.

Corollary 2.3. Suppose, in Lemma 2.2, that $C$ is the direct sum of subspaces, $C=\bigoplus_{i} X_{i}$. Then $C \imath B=\bigoplus_{i}\left(X_{i} \prec B\right)$ and, for each $i$,

$$
X_{i} \prec B=X_{i} \oplus\left[X_{i}, B\right] \oplus\left[X_{i}, B, B\right] \oplus \cdots .
$$

Furthermore, for each $n, \zeta_{n}$ restricts to an isomorphism

$$
[X_{i}, \underbrace{B, \ldots, B}_{n}] \stackrel{\cong}{\longrightarrow} X_{i} \otimes \underbrace{B \otimes \cdots \otimes B}_{n} .
$$

We next consider Schur algebras for the general linear group. Basically, we follow [16], but we adapt the treatment there to allow the underlying field to be finite or infinite. Details omitted in our treatment can be found in [16] or are straightforward modifications of what can be found there. As before, $R$ is either the ring of integers or an arbitrary field.

Let $n$ and $r$ be positive integers. Let $I(n, r)$ be the set of all ordered $r$-tuples $\mathbf{i}=\left(i_{1}, \ldots, i_{r}\right)$ where $i_{1}, \ldots, i_{r} \in\{1, \ldots, n\}$.

Let $A_{R}(n, r)$ be the homogeneous component of degree $r$ in the polynomial ring over $R$ in $n^{2}$ indeterminates $c_{i j}(1 \leqslant i, j \leqslant n)$. Thus $A_{R}(n, r)$ has an $R$-basis consisting of the monomials of degree $r$. For $\mathbf{i}, \mathbf{j} \in I(n, r)$ where $\mathbf{i}=\left(i_{1}, \ldots, i_{r}\right)$, $\mathbf{j}=\left(j_{1}, \ldots, j_{r}\right)$, we write $c_{\mathbf{i}, \mathbf{j}}$ for the monomial $c_{i_{1} j_{1}} \cdots c_{i_{r} j_{r}}$. The $c_{\mathbf{i}, \mathbf{j}}$ are not distinct (when $n, r>1$ ) but they give a basis (with repetitions) of $A_{R}(n, r)$.

Let $S_{R}(n, r)=\operatorname{Hom}_{R}\left(A_{R}(n, r), R\right)$. Thus $S_{R}(n, r)$ has an $R$-basis (with repetitions) consisting of the elements $\xi_{\mathbf{i}, \mathbf{j}}(\mathbf{i}, \mathbf{j} \in I(n, r))$ where $\xi_{\mathbf{i}, \mathbf{j}}\left(c_{\mathbf{i}, \mathbf{j}}\right)=1$ and $\xi_{\mathbf{i}, \mathbf{j}}\left(c_{\mathbf{i}^{\prime}, \mathbf{j}^{\prime}}\right)=0$ if $c_{\mathbf{i}^{\prime}, \mathbf{j}^{\prime}} \neq c_{\mathbf{i}, \mathbf{j}}$. Multiplication in $S_{R}(n, r)$ may be defined as in [16, $\S 2.3]$ : for $\xi, \eta \in S_{R}(n, r)$,

$$
(\xi \eta)\left(c_{\mathbf{i}, \mathbf{j}}\right)=\sum_{\mathbf{k} \in I(n, r)} \xi\left(c_{\mathbf{i}, \mathbf{k}}\right) \eta\left(c_{\mathbf{k}, \mathbf{j}}\right) .
$$

Thus $S_{R}(n, r)$ becomes an associative $R$-algebra with identity element. This is the Schur algebra of degree $r$. As in $[\mathbf{1 6},(2.3 \mathrm{~b})]$ there are equations

$$
\xi_{\mathbf{i}, \mathbf{j}} \xi_{\mathbf{k}, \mathbf{l}}=\sum z_{\mathbf{s}, \mathbf{t}} \xi_{\mathbf{s}, \mathbf{t}}
$$


where the $z_{\mathbf{s}, \mathbf{t}}$ are non-negative integers depending on $\mathbf{i}, \mathbf{j}, \mathbf{k}, \mathbf{l}$ but independent of $R$. When we need to show the role of $R$ we write $\xi_{\mathbf{i}, \mathbf{j}}^{R}$ instead of $\xi_{\mathbf{i}, \mathbf{j}}$. We may identify $S_{R}(n, r)$ with $R \otimes_{\mathbb{Z}} S_{\mathbb{Z}}(n, r)$ by identifying $\xi_{\mathbf{i}, \mathbf{j}}^{R}$ with $1 \otimes \xi_{\mathbf{i}, \mathbf{j}}^{\mathbb{Z}}$ for all $\mathbf{i}, \mathbf{j}$. If $E$ is a field containing $R$ we may regard $S_{R}(n, r)$ as a subring of $S_{E}(n, r)$ by identifying $\xi_{\mathbf{i}, \mathbf{j}}^{R}$ with $\xi_{\mathbf{i}, \mathbf{j}}^{E}$. We may also identify $S_{E}(n, r)$ with $E \otimes_{R} S_{R}(n, r)$.

For $w \in A_{R}(n, r)$ and $g=\left(g_{i j}\right) \in \mathrm{GL}(n, R)$ we write $w(g)$ for the element of $R$ obtained from $w$ by evaluating $c_{i j}$ as $g_{i j}$ for all $i, j$. We write $e(g)$ for the element of $S_{R}(n, r)$ given by $e(g)(w)=w(g)$ for all $w \in A_{R}(n, r)$. Then, as in [16, §2.4], e extends to an algebra homomorphism

$$
e: R \mathrm{GL}(n, R) \longrightarrow S_{R}(n, r)
$$

This is surjective if $R$ is an infinite field, by [16, (2.4b)].

We shall consider right modules for $R \mathrm{GL}(n, R)$ and $S_{R}(n, r)$, whereas $[\mathbf{1 6}]$ has left modules. This leads only to minor, mainly notational, differences. Suppose that $U$ is an $S_{R}(n, r)$-module which is free of finite rank as an $R$-module. Then $U$ may be regarded as an $R \mathrm{GL}(n, R)$-module by means of $e$, and such $R \mathrm{GL}(n, R)$ modules are called polynomial modules of degree $r$. If $U$ has $R$-basis $\left\{u_{1}, \ldots, u_{d}\right\}$ then there are uniquely determined elements $w_{s t}(1 \leqslant s, t \leqslant d)$ of $A_{R}(n, r)$ such that, for all $\xi \in S_{R}(n, r)$ and all $s$,

$$
u_{s} \xi=\sum_{t} \xi\left(w_{s t}\right) u_{t}
$$

These polynomials $w_{s t}$ are called the coefficient polynomials (with respect to the given basis). For all $g \in \mathrm{GL}(n, R)$ we also have

$$
u_{s} g=\sum_{t} w_{s t}(g) u_{t}
$$

If $R=E$, where $E$ is an infinite field, then the coefficient polynomials are uniquely determined by the equations (2.3) and the existence of such polynomials for an $E G L(n, E)$-module $U$ implies that $U$ is a polynomial module of degree $r$.

References for basic facts about polynomial modules are only conveniently available when $R$ is an infinite field. Thus the following result is often useful.

Lemma 2.4. Let $U$ be an $R \mathrm{GL}(n, R)$-module with $R$-basis $\left\{u_{1}, \ldots, u_{d}\right\}$. Let $E$ be an infinite field containing $R$. Then $U$ is a polynomial module of degree $r$ if 
and only if $E \otimes_{R} U$ is a polynomial $E \mathrm{GL}(n, E)$-module which restricts to give $U$, on taking $U \subseteq E \otimes_{R} U$ and $\operatorname{GL}(n, R) \subseteq \mathrm{GL}(n, E)$, and such that the coefficient polynomials for $E \otimes_{R} U$ with respect to $\left\{u_{1}, \ldots, u_{d}\right\}$ belong to $A_{R}(n, r)$.

Proof. (Sketch.) Suppose that $E \otimes_{R} U$ has the given properties with coefficient polynomials $w_{s t} \in A_{R}(n, r)$. By (2.2), $U$ is invariant under the action of $S_{R}(n, r)$. Conversely, if $U$ is an $S_{R}(n, r)$-module with coefficient polynomials $w_{s t}$ then (2.2) holds for $E \otimes_{R} U$ because it holds for $U$.

Let $V$ be a free $R$-module of rank $n$ with basis $\left\{x_{1}, \ldots, x_{n}\right\}$. The identity representation $\mathrm{GL}(n, R) \rightarrow \mathrm{GL}(n, R)$ gives $V$ the structure of the "natural" $R \mathrm{GL}(n, R)$-module. It is easy to check (by Lemma 2.4, for example) that $V$ is an $S_{R}(n, 1)$-module. Indeed the coefficient polynomials are given by $w_{i j}=c_{i j}$ for all $i, j \in\{1, \ldots, n\}$. For every positive integer $r, T^{r}(V), L^{r}(V)$ and $S^{r}(V)$ are $R \mathrm{GL}(n, R)$-modules as we saw earlier in this section. By Lemma 2.4, they are $S_{R}(n, r)$-modules. In $\S 3$ we shall consider $R \mathrm{GL}(n, R)$-modules of the form

$$
S^{m(1)}\left(L^{1}(V)\right) \otimes_{R} S^{m(2)}\left(L^{2}(V)\right) \otimes_{R} \cdots \otimes_{R} S^{m(r)}\left(L^{r}(V)\right),
$$

where $m(1)+2 m(2)+\cdots+r m(r)=r$. Again they are $S_{R}(n, r)$-modules.

From now on in this section we consider only the case $R=F$ where $F$ is a field (finite or infinite). Thus $V$ is an $n$-dimensional vector space over $F$.

For $\mathbf{i} \in I(n, r)$ where $\mathbf{i}=\left(i_{1}, \ldots, i_{r}\right)$, write $x_{\mathbf{i}}=x_{i_{1}} \cdots x_{i_{r}} \in T^{r}(V)$. Thus the elements $x_{\mathbf{i}}$ form a basis of $T^{r}(V)$. The action of $S_{F}(n, r)$ on $T^{r}(V)$ is given by [16, (2.6a)]. Translating to right modules we have

$$
x_{\mathbf{i}} \xi=\sum_{\mathbf{j}} \xi\left(c_{\mathbf{i}, \mathbf{j}}\right) x_{\mathbf{j}},
$$

for all $\xi \in S_{F}(n, r)$.

Let $\Lambda(n, r)$ be the set of all $n$-tuples $\alpha=\left(\alpha_{1}, \ldots, \alpha_{n}\right)$ of non-negative integers satisfying $\alpha_{1}+\cdots+\alpha_{n}=r$. For each such $\alpha$ take $\mathbf{i} \in I(n, r)$ so that $c_{11}^{\alpha_{1}} c_{22}^{\alpha_{2}} \cdots c_{n n}^{\alpha_{n}}=c_{\mathbf{i}, \mathbf{i}}$ and write $\xi_{\alpha}=\xi_{\mathbf{i}, \mathbf{i}}$. Thus (see [16, $\S 2.3$ and $\left.\S 3.2\right]$ ) the elements $\xi_{\alpha}$ are pairwise orthogonal idempotents of $S_{F}(n, r)$ which sum to the identity. It follows that if $W$ is any $S_{F}(n, r)$-module then

$$
W=\bigoplus_{\alpha \in \Lambda(n, r)} W \xi_{\alpha},
$$


where the $W \xi_{\alpha}$ are subspaces, called weight spaces.

The multidegree of a monomial $x_{i_{1}} \cdots x_{i_{r}}$ of $T^{r}(V)$ is defined to be the element $\alpha=\left(\alpha_{1}, \ldots, \alpha_{n}\right)$ of $\Lambda(n, r)$ such that, for $j=1, \ldots, n, \alpha_{j}$ is the number of values of $t$ such that $i_{t}=j$. An element of $T^{r}(V)$ is said to be multihomogeneous if it is a linear combination of monomials of the same multidegree.

By (2.4), the action of $\xi_{\alpha}$ on $T^{r}(V)$ is given by

$$
\left(x_{i_{1}} \cdots x_{i_{r}}\right) \xi_{\alpha}= \begin{cases}x_{i_{1}} \cdots x_{i_{r}} & \text { if } x_{i_{1}} \cdots x_{i_{r}} \text { has multidegree } \alpha \\ 0 & \text { otherwise. }\end{cases}
$$

Thus, if $W$ is an $S_{F}(n, r)$-submodule of $T^{r}(V)$, the weight space $W \xi_{\alpha}$, as in (2.5), consists of multihomogeneous elements of multidegree $\alpha$.

We shall need to consider $S_{F}(n, r)$-submodules of $T^{r}(V)$ as $n$ varies. Thus, let $V^{(\omega)}$ be a vector space over $F$ with countably infinite basis $\left\{x_{1}, x_{2}, \ldots\right\}$. For each positive integer $n$, let $V^{(n)}$ be the subspace with basis $\left\{x_{1}, \ldots, x_{n}\right\}$ and identify $T\left(V^{(n)}\right)$ with a subalgebra of $T\left(V^{(\omega)}\right)$ in the obvious way. Also, for each $n$, regard $V^{(n)}$ as the natural $F \mathrm{GL}(n, F)$-module. Thus $T^{r}\left(V^{(n)}\right)$ is an $S_{F}(n, r)$-module.

Let $n_{1}$ and $n_{2}$ be positive integers with $n_{1} \leqslant n_{2}$. Thus $I\left(n_{1}, r\right) \subseteq I\left(n_{2}, r\right)$. For $\mathbf{i}, \mathbf{j} \in I\left(n_{1}, r\right)$ we may identify $c_{\mathbf{i}, \mathbf{j}}$ in $A_{F}\left(n_{1}, r\right)$ with $c_{\mathbf{i}, \mathbf{j}}$ in $A_{F}\left(n_{2}, r\right)$ to make $A_{F}\left(n_{1}, r\right)$ a subspace of $A_{F}\left(n_{2}, r\right)$. Similarly, by identifying elements $\xi_{\mathbf{i}, \mathbf{j}}$, we make $S_{F}\left(n_{1}, r\right)$ a subspace of $S_{F}\left(n_{2}, r\right)$. It is easy to verify that $S_{F}\left(n_{1}, r\right)$ is a subalgebra of $S_{F}\left(n_{2}, r\right)$ (though the identity elements are different if $n_{1}<n_{2}$ ). We may also take $\Lambda\left(n_{1}, r\right) \subseteq \Lambda\left(n_{2}, r\right)$ by identifying $\left(\alpha_{1}, \ldots, \alpha_{n_{1}}\right)$ with $\left(\alpha_{1}, \ldots, \alpha_{n_{1}}, 0, \ldots, 0\right)$.

Let $W$ be an $S_{F}\left(n_{2}, r\right)$-submodule of $T^{r}\left(V^{\left(n_{2}\right)}\right)$. Thus $W=\bigoplus_{\alpha \in \Lambda\left(n_{2}, r\right)} W \xi_{\alpha}$. The truncation function $d_{n_{2}, n_{1}}$ of $[\mathbf{1 6}, \S 6.5]$ gives

$$
d_{n_{2}, n_{1}}(W)=\bigoplus_{\alpha \in \Lambda\left(n_{1}, r\right)} W \xi_{\alpha}
$$

and $d_{n_{2}, n_{1}}(W)$ is an $S_{F}\left(n_{1}, r\right)$-submodule of $T^{r}\left(V^{\left(n_{1}\right)}\right)$. Another point of view is useful. Let $\delta_{n_{2}, n_{1}}: T\left(V^{\left(n_{2}\right)}\right) \rightarrow T\left(V^{\left(n_{1}\right)}\right)$ be the algebra homomorphism defined by

$$
\delta_{n_{2}, n_{1}}\left(x_{i}\right)= \begin{cases}x_{i} & \text { for } i \in\left\{1, \ldots, n_{1}\right\} \\ 0 & \text { for } i \in\left\{n_{1}+1, \ldots, n_{2}\right\}\end{cases}
$$

Then it is easily verified, by (2.6), that

$$
\delta_{n_{2}, n_{1}}(W)=d_{n_{2}, n_{1}}(W)=W \cap T\left(V^{\left(n_{1}\right)}\right) .
$$


Let $\left\{W^{(n)}: n \in \mathbb{N}\right\}$ be a family of subspaces of $T\left(V^{(\omega)}\right)$. (We write $\mathbb{N}$ for the set of all positive integers.) We say that $\left\{W^{(n)}\right\}$ is a uniform family of modules of degree $r$ if $W^{(n)}$ is an $S_{F}(n, r)$-submodule of $T^{r}\left(V^{(n)}\right)$ for all $n$ and $\delta_{n_{2}, n_{1}}\left(W^{\left(n_{2}\right)}\right)=W^{\left(n_{1}\right)}$ for all $n_{1}, n_{2}$ with $n_{2} \geqslant n_{1}$. For example, the family $\left\{T^{r}\left(V^{(n)}\right)\right\}$ is a uniform family of modules of degree $r$.

Lemma 2.5. Let $W$ be an $S_{F}(r, r)$-submodule of $T^{r}\left(V^{(r)}\right)$. Then there exists a unique uniform family $\left\{W^{(n)}\right\}$ of modules of degree $r$ such that $W^{(r)}=W$.

Proof. Suppose that $n \geqslant r$. We let the symmetric group $\operatorname{Sym}(n)$ act on the right as a group of automorphisms of $T\left(V^{(n)}\right)$ by extension of its right permutation action on $\left\{x_{1}, \ldots, x_{n}\right\}$. For $\pi \in \operatorname{Sym}(n)$ and $\mathbf{i} \in I(n, r)$, where $\mathbf{i}=\left(i_{1}, \ldots, i_{r}\right)$, we write $\mathbf{i} \pi=\left(i_{1} \pi, \ldots, i_{r} \pi\right)$. Also, we identify $\pi$ with the corresponding permutation matrix in $\operatorname{GL}(n, F)$ and write $\tilde{\pi}$ for the element of $S_{F}(n, r)$ obtained from $\pi$ by the homomorphism (2.1). It is straightforward to verify that in $S_{F}(n, r)$ we have

$$
\tilde{\pi} \xi_{\mathbf{i}, \mathbf{j}}=\xi_{\mathbf{i} \pi^{-1}, \mathbf{j}} \text { and } \xi_{\mathbf{i}, \mathbf{j}} \tilde{\pi}=\xi_{\mathbf{i}, \mathbf{j} \pi}
$$

for all $\mathbf{i}, \mathbf{j} \in I(n, r)$.

We shall show that $\sum_{\pi \in \operatorname{Sym}(n)} W \pi$ is an $S_{F}(n, r)$-submodule of $T^{r}\left(V^{(n)}\right)$. Let $\sigma \in \operatorname{Sym}(n)$ and $\mathbf{i}, \mathbf{j} \in I(n, r)$. Choose $\pi \in \operatorname{Sym}(n)$ so that $\mathbf{j} \pi^{-1} \in I(r, r)$. By (2.7),

$$
\tilde{\sigma} \xi_{\mathbf{i}, \mathbf{j}}=\xi_{\mathbf{i} \sigma^{-1}, \mathbf{j}}=\xi_{\mathbf{i} \sigma^{-1}, \mathbf{j} \pi^{-1}} \tilde{\pi}
$$

For $w \in W, w \xi_{\mathbf{i} \sigma^{-1}, \mathbf{j} \pi^{-1}}=0$ if $\mathbf{i} \sigma^{-1} \notin I(r, r)$, by (2.4). However, if $\mathbf{i} \sigma^{-1} \in I(r, r)$, then $\xi_{\mathbf{i} \sigma^{-1}, \mathbf{j} \pi^{-1}} \in S_{F}(r, r)$, so $w \xi_{\mathbf{i} \sigma^{-1}, \mathbf{j} \pi^{-1}} \in W$. Thus, by $(2.8),(W \sigma) \xi_{\mathbf{i}, \mathbf{j}} \subseteq W \pi$. Hence $\sum_{\pi \in \operatorname{Sym}(n)} W \pi$ is a submodule of $T^{r}\left(V^{(n)}\right)$.

For $n \geqslant r$ we define $W^{(n)}=\sum_{\pi \in \operatorname{Sym}(n)} W \pi$ and for $n<r$ we define $W^{(n)}=\delta_{r, n}(W)$. Then it is straightforward to verify that the family $\left\{W^{(n)}\right\}$ is unique with the required properties.

LEMMA 2.6. Let $W_{1}$ and $W_{2}$ be submodules of $T^{r}\left(V^{(r)}\right)$ which span their direct sum, and let $\left\{W_{1}^{(n)}\right\},\left\{W_{2}^{(n)}\right\}$ and $\left\{\left(W_{1} \oplus W_{2}\right)^{(n)}\right\}$ be the uniform families given by Lemma 2.5. Then, for all $n$,

$$
\left(W_{1} \oplus W_{2}\right)^{(n)}=W_{1}^{(n)} \oplus W_{2}^{(n)}
$$


Proof. This is straightforward.

Lemma 2.7. Suppose that $\left\{W_{1}^{(n)}\right\}, \ldots,\left\{W_{m}^{(n)}\right\}$ are uniform families of modules of degrees $r_{1}, \ldots, r_{m}$, respectively. Then $\left\{\left[W_{1}^{(n)}, W_{2}^{(n)}, \ldots, W_{m}^{(n)}\right]\right\}$ is a uniform family of modules of degree $r_{1}+\cdots+r_{m}$.

Proof. Let $r=r_{1}+\cdots+r_{m}$. By Lemma $2.4,\left[W_{1}^{(n)}, \ldots, W_{m}^{(n)}\right]$ is an $S_{F}(n, r)$ submodule of $T^{r}\left(V^{(n)}\right)$ for all $n$. Suppose that $n_{1} \leqslant n_{2}$. Then, since, $\delta_{n_{2}, n_{1}}$ is an algebra homomorphism,

$$
\begin{aligned}
\delta_{n_{2}, n_{1}}\left(\left[W_{1}^{\left(n_{2}\right)}, \ldots, W_{m}^{\left(n_{2}\right)}\right]\right) & =\left[\delta_{n_{2}, n_{1}}\left(W_{1}^{\left(n_{2}\right)}\right), \ldots, \delta_{n_{2}, n_{1}}\left(W_{m}^{\left(n_{2}\right)}\right)\right] \\
& =\left[W_{1}^{\left(n_{1}\right)}, \ldots, W_{m}^{\left(n_{1}\right)}\right] .
\end{aligned}
$$

Thus the family is uniform.

In particular, $\left\{L^{r}\left(V^{(n)}\right): n \in \mathbb{N}\right\}$ is a uniform family of modules of degree $r$.

We shall wish to consider arbitrary fields, possibly finite. However, it is often convenient to pass to the infinite case, so we require some results on field extensions.

LEMma 2.8. Let $F$ be a field and $E$ an extension field of $F$. Let $A$ be an $F$-algebra and $U$ a finite-dimensional A-module. If $E \otimes_{F} U$ is injective, as an $E \otimes_{F} A$-module, then $U$ is injective. If $E \otimes_{F} U$ is projective then $U$ is projective.

Proof. Suppose that $E \otimes U$ is injective as an $E \otimes A$-module. Then, by $[\mathbf{1 1}$, Proposition II.6.2a], $E \otimes U$ is injective as an $A$-module. But $U$ is a direct summand of $E \otimes U$ as an $A$-module. Hence $U$ is injective. The result for projective modules is proved in the same way, using [11, Proposition II.6.2].

As above, we consider $V^{(n)}$ over an arbitrary field $F$.

Lemma 2.9. Suppose that $r \leqslant n$ and let $U$ be an $S_{F}(n, r)$-module which is isomorphic to a direct summand of $T^{r}\left(V^{(n)}\right)$. Then $U$ is injective and projective as an $S_{F}(n, r)$-module. 
Proof. Let $E$ be an infinite extension field of $F$. Thus $E \otimes U$ is isomorphic to a direct summand of $T^{r}\left(E \otimes V^{(n)}\right)$. Since $r \leqslant n, T^{r}\left(E \otimes V^{(n)}\right)$ is injective as an $S_{E}(n, r)$-module, by [12, (3.4) Lemma]. However, by [16, Example 1 after $(2.7 \mathrm{e})], T^{r}\left(E \otimes V^{(n)}\right)$ is isomorphic to its contravariant dual. Thus $T^{r}\left(E \otimes V^{(n)}\right)$ is projective. It follows that $E \otimes U$ is injective and projective. Therefore, by Lemma 2.8, $U$ is injective and projective.

For positive integers $n$ and $r$, let $\mathcal{T}_{r}^{(n)}$ denote the class of all finite-dimensional $S_{F}(n, r)$-modules that are direct sums of modules each of which is isomorphic to a direct summand of $T^{r}\left(V^{(n)}\right)$.

LEMMA 2.10. Let $\left\{W^{(n)}\right\}$ be a uniform family of modules of degree $r$ such that $W^{(r)} \in \mathcal{T}_{r}^{(r)}$. Then, for all $n, W^{(n)}$ is a direct summand of $T^{r}\left(V^{(n)}\right)$.

Proof. By Lemma 2.9, $W^{(r)}$ is injective. Therefore, since $W^{(r)}$ is a submodule of $T^{r}\left(V^{(r)}\right)$, it is a direct summand of $T^{r}\left(V^{(r)}\right)$. It follows, by Lemma 2.6, that $W^{(n)}$ is a direct summand of $T^{r}\left(V^{(n)}\right)$ for all $n$.

\section{Filtrations of tensor powers}

As in $\S 2$, we first work over a ring $R$ which is either the ring of integers or an arbitrary field. Let $V$ be a free $R$-module and let $r$ be a positive integer.

By a composition of $r$ we mean a sequence $\lambda=\left(\lambda_{1}, \ldots, \lambda_{l}\right)$ of positive integers $\lambda_{1}, \ldots, \lambda_{l}$ satisfying $\lambda_{1}+\cdots+\lambda_{l}=r$. If we also have $\lambda_{1} \geqslant \cdots \geqslant \lambda_{l}$ then, as usual, $\lambda$ is called a partition of $r$. If $\mu=\left(\mu_{1}, \ldots, \mu_{l}\right)$ is a composition of $r$ and we put $\mu_{1}, \ldots, \mu_{l}$ into non-increasing order we obtain a partition of $r$, which we call the partition associated to $\mu$. We write $\operatorname{Part}(r)$ for the set of all partitions of $r$.

If $\lambda, \theta \in \operatorname{Part}(r)$, where $\lambda=\left(\lambda_{1}, \ldots, \lambda_{k}\right)$ and $\theta=\left(\theta_{1}, \ldots, \theta_{l}\right)$, we say that $\lambda$ is a refinement of $\theta$ if there is a function $f:\{1, \ldots, k\} \rightarrow\{1, \ldots, l\}$ such that $\theta_{j}=\sum_{i: f(i)=j} \lambda_{i}$ for $j=1, \ldots, l$. For example, $(3,2,2,2,1)$ is a refinement of $(4,3,3)$. The relation of refinement is a partial order on $\operatorname{Part}(r)$, and this partial order plays a key role in the study of filtrations of tensor powers. However, it is 
more convenient here to work with a total order $\leqslant{ }^{*}$ which extends refinement. We use the "lexicographic" order in which

$$
\left(\lambda_{1}, \lambda_{2}, \ldots, \lambda_{k}\right)<^{*}\left(\theta_{1}, \theta_{2}, \ldots, \theta_{l}\right)
$$

if there exists $i$ such that $\lambda_{i}<\theta_{i}$ but $\lambda_{j}=\theta_{j}$ for all $j<i$. Note that if $\lambda$ is a refinement of $\theta$ then $\lambda \leqslant * \theta$. Note also that the smallest partition is $(1,1, \ldots, 1)$, written as $\left(1^{r}\right)$, and the largest partition is $(r)$.

Our main results are stated in terms of the lexicographic order $\leqslant^{*}$, but similar results apply for any total order which extends refinement, and more complicated versions of the results could be stated in terms of refinement itself.

Let $\mathcal{X}_{r}$ be the set of all elements of $T^{r}(V)$ of the form $b_{1} b_{2} \cdots b_{l}$ ( $l$ arbitrary) where each $b_{i}$ belongs to $L^{\mu_{i}}(V)$ for some positive integer $\mu_{i}$. Thus $\mu_{i}=\operatorname{deg} b_{i}$ for all $i$ and $\left(\mu_{1}, \ldots, \mu_{l}\right)$ is a composition of $r$. For each partition $\lambda$ of $r$ let $\mathcal{X}_{\lambda}$ denote the set of all such elements $b_{1} b_{2} \cdots b_{l}$ where $\left(\operatorname{deg} b_{1}, \ldots, \operatorname{deg} b_{l}\right)$ has $\lambda$ as its associated partition.

For each $\lambda$, let $W_{\lambda}$ be the $R$-submodule of $T^{r}(V)$ spanned by all $\mathcal{X}_{\theta}$ with $\lambda \leqslant * \theta$. For each $\lambda$ such that $\lambda \neq(r)$ let $\lambda+$ be the partition of $r$ which is next bigger than $\lambda$. Thus we have the filtration

$$
T^{r}(V)=W_{\left(1^{r}\right)} \geqslant \cdots \geqslant W_{\lambda} \geqslant W_{\lambda+} \geqslant \cdots \geqslant W_{(r)} \geqslant 0 .
$$

We call this the PBW-filtration of $T^{r}(V)$. (As we shall see it is closely connected with the Poincaré-Birkhoff-Witt Theorem.) It is convenient to define $W_{(r)+}=0$. Thus, for all $\lambda, \mathcal{X}_{\lambda}$ spans $W_{\lambda}$ modulo $W_{\lambda+}$.

The following result is well known and easy to prove using the identity $b_{i} b_{j}=b_{j} b_{i}+\left[b_{i}, b_{j}\right]$.

LEMMA 3.1. Let $\mu=\left(\mu_{1}, \ldots, \mu_{l}\right)$ be a composition of $r$ with associated partition $\lambda$. For $i=1, \ldots, l$, let $b_{i} \in L^{\mu_{i}}(V)$, and let $\pi \in \operatorname{Sym}(l)$ : thus $b_{1} b_{2} \cdots b_{l}$ and $b_{1 \pi} b_{2 \pi} \cdots b_{l \pi}$ belong to $\mathcal{X}_{\lambda}$. We have

$$
b_{1} b_{2} \cdots b_{l}+W_{\lambda+}=b_{1 \pi} b_{2 \pi} \cdots b_{l \pi}+W_{\lambda+} .
$$


For each positive integer $i$ choose an $R$-basis $\mathcal{A}_{i}$ of $L^{i}(V)$. The union of these is an $R$-basis $\mathcal{A}$ of $L(V)$. Take any total ordering $\preccurlyeq$ of $\mathcal{A}$. Then, by the PoincaréBirkhoff-Witt Theorem (see [18] for the case of a field and [2] for general $R$ ), $T(V)$ has an $R$-basis $\mathcal{F}$ consisting of all elements of the form

$$
a_{1} a_{2} \cdots a_{l} \quad\left(l \geqslant 0, a_{1}, \ldots, a_{l} \in \mathcal{A}, a_{1} \preccurlyeq a_{2} \preccurlyeq \cdots \preccurlyeq a_{l}\right) .
$$

We call $\mathcal{F}$ a PBW-basis of $T(V)$. Note that the elements (3.1) of $\mathcal{F}$ are distinct as written. We write $\mathcal{F}_{r}$ for the set of all elements (3.1) of degree $r$. Also, for each $\lambda \in \operatorname{Part}(r)$, we write $\mathcal{F}_{\lambda}$ for the set of elements (3.1) in $\mathcal{F}_{r}$ such that the composition $\left(\operatorname{deg} a_{1}, \ldots, \operatorname{deg} a_{l}\right)$ has $\lambda$ as associated partition. Clearly, $\mathcal{F}_{r}$ is an $R$-basis of $T^{r}(V)$, called a PBW-basis of $T^{r}(V)$. Also, it is easy to verify, by Lemma 3.1 and reverse induction on $\lambda$ (starting with $\lambda=(r)$ ), that the elements of $\mathcal{F}_{\lambda}$ taken modulo $W_{\lambda+}$ form an $R$-basis of $W_{\lambda} / W_{\lambda+}$. (It follows that $W_{\lambda}>W_{\lambda+}$ provided that $\operatorname{dim} V>1$.)

Suppose that $V$ is an $R G$-module for some group $G$. Let $\lambda \in \operatorname{Part}(r)$ and write $\lambda$ in the form

$$
\lambda=\left(r^{m(r)},(r-1)^{m(r-1)}, \ldots, 1^{m(1)}\right),
$$

where $m(r), \ldots, m(1)$ are non-negative integers. Then we define an $R G$-module $L^{\lambda}(V)$ by

$$
L^{\lambda}(V)=S^{m(1)}\left(L^{1}(V)\right) \otimes_{R} S^{m(2)}\left(L^{2}(V)\right) \otimes_{R} \cdots \otimes_{R} S^{m(r)}\left(L^{r}(V)\right) .
$$

This is called the higher Lie module corresponding to $\lambda$.

The following result is essentially well known: see, for example, $[\mathbf{1 7}$, Lemma 3.3]. However, since we have no fully appropriate reference we give a sketch proof.

LEMmA 3.2. Suppose that the free $R$-module $V$ is an $R G$-module for some group $G$. Then, for each $\lambda \in \operatorname{Part}(r), W_{\lambda}$ is an $R G$-submodule of $T^{r}(V)$ and $W_{\lambda} / W_{\lambda+} \cong L^{\lambda}(V)$.

Proof. (Sketch.) It is easily verified by reverse induction on $\lambda$ that $W_{\lambda}$ is an $R G$-submodule of $T^{r}(V)$. In the notation used above, choose the ordering of $\mathcal{A}$ in any way so that all elements of $\mathcal{A}_{i}$ are smaller than all elements of $\mathcal{A}_{j}$ whenever 
$i<j$. The basis $\left\{w+W_{\lambda+}: w \in \mathcal{F}_{\lambda}\right\}$ of $W_{\lambda} / W_{\lambda+}$ consists of all elements of the form

$$
a_{1}^{(1)} \cdots a_{1}^{(m(1))} a_{2}^{(1)} \cdots a_{2}^{(m(2))} \cdots a_{r}^{(1)} \cdots a_{r}^{(m(r))}+W_{\lambda+}
$$

where the factors are in non-decreasing order and $a_{i}^{(1)}, \ldots, a_{i}^{(m(i))} \in \mathcal{A}_{i}$ for $i=1, \ldots, r$. There is a corresponding basis of $L^{\lambda}(V)$ consisting of the elements

$$
a_{1}^{(1)} \cdots a_{1}^{(m(1))} \otimes a_{2}^{(1)} \cdots a_{2}^{(m(2))} \otimes \cdots \otimes a_{r}^{(1)} \cdots a_{r}^{(m(r))}
$$

It is easy to verify, by Lemma 3.1, that the $R$-module isomorphism $\alpha$ that takes basis element to corresponding basis element is an $R G$-module isomorphism.

In particular, $W_{\left(1^{r}\right)} / W_{\left(1^{r}\right)+} \cong S^{r}(V)$ and $W_{(r)} \cong L^{r}(V)$.

If $V$ has finite rank $n$ then the isomorphism of Lemma 3.2 is an $S_{R}(n, r)$-module isomorphism. This is clear if $R$ is an infinite field (because then the homomorphism (2.1) is surjective). In the general case, let $E$ be an infinite field containing $R$. By Lemma 3.2, $W_{\lambda}$ is an $R \mathrm{GL}(n, R)$-submodule of $T^{r}(V)$. We regard $T^{r}(V)$ as a subset of $T^{r}(E \otimes V)$ and identify this with $E \otimes T^{r}(V)$. It is easily verified that $E \otimes W_{\lambda}$ is the subspace corresponding to $\lambda$ in the PBW-filtration of $T^{r}(E \otimes V)$. Thus we may verify by Lemma 2.4 that $W_{\lambda}$ is an $S_{R}(n, r)$-submodule of $T^{r}(V)$. Let $\alpha$ be the $R$-module isomorphism from $W_{\lambda} / W_{\lambda+}$ to $L^{\lambda}(V)$ described in the proof of Lemma 3.2. Upon extension to $E, \alpha$ yields an $S_{E}(n, r)$-module isomorphism, by the case first considered. Therefore $\alpha$ is an $S_{R}(n, r)$-module isomorphism.

We shall make use of Solomon's descent algebra. For definitions and further details see $[\mathbf{1 4}, \S 2]$ and the works cited there. As well as results from $[\mathbf{1 4}]$ we shall use a theorem of Garsia and Reutenauer [15]. Our notation is similar to that in $[\mathbf{1 4}, \S 2]$. Let $D_{r}$ be the descent algebra of $\operatorname{Sym}(r)$ over the integers. Thus $D_{r}$ is a subalgebra of $\mathbb{Z} \operatorname{Sym}(r)$ and $D_{r}$ has a $\mathbb{Z}$-basis consisting of certain elements $X^{\nu}$ of $\mathbb{Z} \operatorname{Sym}(r)$ indexed by the compositions $\nu$ of $r$. (In the notation of $[\mathbf{1 5}], X^{\nu}$ would be written as $B_{\nu}$.) The elements $X^{\nu}$ can be interpreted as elements of $R \operatorname{Sym}(r)$ and these elements form an $R$-basis for an $R$-subalgebra $D_{r, R}$ of $R \operatorname{Sym}(r)$.

For each composition $\nu$ of $r$, where $\nu=\left(\nu_{1}, \ldots, \nu_{k}\right)$, we write $\phi^{\nu}$ for the corresponding Young character of $\operatorname{Sym}(r)$. This is the character (in characteristic $0)$ induced from the trivial character of the group $\operatorname{Sym}\left(\nu_{1}\right) \times \cdots \times \operatorname{Sym}\left(\nu_{k}\right)$ regarded 
as a subgroup of $\operatorname{Sym}(r)$ in the obvious way. The $\mathbb{Z}$-span of the Young characters is a subring of the ring of all class functions $\operatorname{Sym}(r) \rightarrow \mathbb{Z}$. For each $\nu$, define $\phi^{\nu, R}: \operatorname{Sym}(r) \rightarrow R$ by $\phi^{\nu, R}(\sigma)=\phi^{\nu}(\sigma) 1_{R}$ for all $\sigma \in \operatorname{Sym}(r)$. Thus the $R$-span of the $\phi^{\nu, R}$ is a subalgebra $C_{r, R}$ of the $R$-algebra of all class functions $\operatorname{Sym}(r) \rightarrow R$. For $\xi \in C_{r, R}$ and $\lambda \in \operatorname{Part}(r)$ we write $\xi(\lambda)$ for the value of $\xi$ at the conjugacy class of $\operatorname{Sym}(r)$ indexed by $\lambda$. By the same proof as for [14, Theorem 3], there is a surjective homomorphism of algebras $c_{r, R}: D_{r, R} \rightarrow C_{r, R}$ satisfying $c_{r, R}\left(X^{\nu}\right)=\phi^{\nu, R}$ for all compositions $\nu$ of $r$.

The symmetric group $\operatorname{Sym}(r)$ has a left action on $T^{r}(V)$ by "place permutations". This action is given by $\sigma\left(v_{1} v_{2} \cdots v_{r}\right)=v_{1 \sigma} v_{2 \sigma} \cdots v_{r \sigma}$ for all $\sigma \in \operatorname{Sym}(r)$, $v_{1}, \ldots, v_{r} \in V$. Thus $T^{r}(V)$ is an $R \operatorname{Sym}(r)$-module and hence a $D_{r, R}$-module.

Proposition 3.3. For each partition $\lambda$ of $r, W_{\lambda}$ is a $D_{r, R}$-submodule of $T^{r}(V)$. For all $X \in D_{r, R}$, the action of $X$ on $W_{\lambda} / W_{\lambda+}$ is given by multiplication by the element $\left(c_{r, R}(X)\right)(\lambda)$ of $R$.

Proof. Let $\mu$ and $\nu$ be compositions of $r$, where $\mu=\left(\mu_{1}, \ldots, \mu_{l}\right)$ and $\nu=\left(\nu_{1}, \ldots, \nu_{k}\right)$. Let $Q(\nu, \mu)$ be the set of all $k$-tuples $\left(I_{1}, \ldots, I_{k}\right)$ where $I_{1}, \ldots, I_{k}$ are subsets of $\{1, \ldots, l\}$ such that $\{1, \ldots, l\}$ is the disjoint union of $I_{1}, \ldots, I_{k}$ and $\nu_{j}=\sum_{i \in I_{j}} \mu_{i}$ for $j=1, \ldots, k$. It is easy to verify that $|Q(\nu, \mu)|$ is the number of $k \times l$ matrices of non-negative integers such that (the $l$-tuple formed by) the sum of the rows is $\mu$, the sum of the columns is $\nu$ and each column contains a unique non-zero entry. Therefore, by [1, (12)],

$$
|Q(\nu, \mu)|=\phi^{\nu}(\lambda)
$$

where $\lambda$ is the partition associated to $\mu$.

For each $q=\left(I_{1}, \ldots, I_{k}\right) \in Q(\nu, \mu)$ let $\pi_{q}$ be the permutation of $\{1, \ldots, l\}$ such that $\left(1 \pi_{q}, 2 \pi_{q}, \ldots, l \pi_{q}\right)$ consists of the elements of $I_{1}$ in increasing order followed by the elements of $I_{2}$ in increasing order, and so on. For $i=1, \ldots, l$, let $b_{i} \in L^{\mu_{i}}(V)$. Then, by [15, Theorem 2.1],

$$
X^{\nu}\left(b_{1} b_{2} \cdots b_{l}\right)=\sum_{q \in Q(\nu, \mu)} b_{1 \pi_{q}} b_{2 \pi_{q}} \cdots b_{l \pi_{q}}
$$


Note that this result, as stated in [15], applies only to the case where $R=\mathbb{Q}$ and $V$ has finite rank. However, since (3.3) involves only finitely many elements of $V$, the same result holds for $V$ of infinite rank. From the result over $\mathbb{Q}$ we can deduce the result over $\mathbb{Z}$ because, if $V$ is a free $\mathbb{Z}$-module, there are natural embeddings of $T(V)$ into $T(\mathbb{Q} \otimes V)$ and of $D_{r}$ into $D_{r, \mathbb{Q}}$. In the case where $R$ is an arbitrary field, let $V$ have basis $\left\{x_{1}, x_{2}, \ldots\right\}$. Both sides of (3.3) are linear in each term $b_{i}$. Thus it suffices to prove (3.3) in the case where each $b_{i}$ is a Lie monomial in $x_{1}, x_{2}, \ldots$ The calculation of $X^{\nu}\left(b_{1} b_{2} \cdots b_{l}\right)$ is then the same as it is over $\mathbb{Z}$ followed by reduction modulo the characteristic of $R$. Thus (3.3) holds in all cases.

Let $\lambda \in \operatorname{Part}(r)$. Then, by (3.3), we have $X^{\nu} u \in W_{\lambda}$ for all $u \in \mathcal{X}_{\lambda}$ and all compositions $\nu$ of $r$. Thus $X u \in W_{\lambda}$ for all $X \in D_{r, R}$. It follows that each $W_{\lambda}$ is a $D_{r, R^{-}}$submodule of $T^{r}(V)$. Hence $W_{\lambda} / W_{\lambda+}$ is a $D_{r, R^{-}}$module. Let $b_{1} b_{2} \cdots b_{l} \in \mathcal{X}_{\lambda}$, with composition $\mu=\left(\operatorname{deg} b_{1}, \ldots, \operatorname{deg} b_{l}\right)$, and let $\nu$ be any composition of $r$. By (3.3) and Lemma 3.1,

$$
X^{\nu}\left(b_{1} b_{2} \cdots b_{l}\right)+W_{\lambda+}=|Q(\nu, \mu)| b_{1} b_{2} \cdots b_{l}+W_{\lambda+} .
$$

Thus, by (3.2), the action of $X^{\nu}$ on $W_{\lambda} / W_{\lambda+}$ is multiplication by the scalar $\phi^{\nu}(\lambda) 1_{R}$. By the definition of $c_{r, R}$, this scalar is $\left(c_{r, R}\left(X^{\nu}\right)\right)(\lambda)$. This holds for all $\nu$, giving the required result.

From now on in this section we assume that $R=F$, where $F$ is a field of prime characteristic $p$. If $\lambda, \theta \in \operatorname{Part}(r)$ and $\sigma_{\lambda}$ and $\sigma_{\theta}$ are elements of $\operatorname{Sym}(r)$ in the conjugacy classes corresponding to $\lambda$ and $\theta$, respectively, then, as in $[\mathbf{1 4}, \S 2]$, we say that $\lambda$ and $\theta$ are $p$-equivalent if $\sigma_{\lambda}^{p^{s}}$ is conjugate to $\sigma_{\theta}^{p^{s}}$ for some positive integer $s$. This gives an equivalence relation on $\operatorname{Part}(r)$. By [14, Proposition 5], for all $\xi \in C_{r, F}$ we have $\xi(\lambda)=\xi(\theta)$ whenever $\lambda$ and $\theta$ are $p$-equivalent.

Let $\mathcal{J}$ be the set of all $p$-equivalence classes of partitions of $r$. As shown in $[\mathbf{1 4}$, Corollary 6], the identity element 1 of $D_{r, F}$ can be written as $1=\sum_{J \in \mathcal{J}} e_{J}$, where the $e_{J}$ are pairwise-orthogonal primitive idempotents in $D_{r, F}$ such that $c_{r, F}\left(e_{J}\right)$ is given by

$$
\left(c_{r, F}\left(e_{J}\right)\right)(\lambda)= \begin{cases}1 & \text { if } \lambda \in J \\ 0 & \text { if } \lambda \notin J\end{cases}
$$


Therefore, by Proposition 3.3, $e_{J}$ acts on $W_{\lambda} / W_{\lambda+}$ as the identity if $\lambda \in J$ and as zero otherwise. Thus

$$
e_{J} W_{\lambda}+W_{\lambda+}= \begin{cases}W_{\lambda} & \text { if } \lambda \in J \\ W_{\lambda+} & \text { if } \lambda \notin J .\end{cases}
$$

Also,

$$
e_{J} w+W_{\lambda+}=w+W_{\lambda+} \text { for all } w \in W_{\lambda} \text {, where } \lambda \in J .
$$

Clearly we obtain a chain of subspaces

$$
e_{J} T^{r}(V)=e_{J} W_{\left(1^{r}\right)} \geqslant \cdots \geqslant e_{J} W_{\lambda} \geqslant e_{J} W_{\lambda+} \geqslant \cdots \geqslant e_{J} W_{(r)} \geqslant 0
$$

Note that, since $e_{J}$ is an idempotent, we have $e_{J} W_{\lambda} \cap W_{\lambda+}=e_{J} W_{\lambda+}$. Thus $e_{J} W_{\lambda} / e_{J} W_{\lambda+} \cong\left(e_{J} W_{\lambda}+W_{\lambda+}\right) / W_{\lambda+}$ and we have

$$
e_{J} W_{\lambda} / e_{J} W_{\lambda+} \cong W_{\lambda} / W_{\lambda+} \text { if } \lambda \in J
$$

while

$$
e_{J} W_{\lambda} / e_{J} W_{\lambda+}=0 \text { if } \lambda \notin J
$$

Therefore, if $J=\{\lambda(1), \ldots, \lambda(s)\}$ with $\lambda(1)<^{*} \cdots<^{*} \lambda(s)$, we may write the chain (3.5) in the form

$$
e_{J} T^{r}(V)=U_{1} \geqslant U_{2} \geqslant \cdots \geqslant U_{s} \geqslant U_{s+1}=0,
$$

where $U_{j} / U_{j+1} \cong W_{\lambda(j)} / W_{\lambda(j)+}$ for $j=1, \ldots, s$.

Suppose that $G$ is a group and $V$ is an $F G$-module. It is well known and easy to verify that the actions of $\operatorname{Sym}(r)$ and $G$ on $T^{r}(V)$ commute. It follows that $e_{J} T^{r}(V)$ is an $F G$-submodule of $T^{r}(V)$ and the function $\phi_{J}: T^{r}(V) \rightarrow e_{J} T^{r}(V)$ given by $w \mapsto e_{J} w$ for all $w \in T^{r}(V)$ is a homomorphism of $F G$-modules. By the properties of the idempotents $e_{J}$ we see that $T^{r}(V)$ is the direct sum of the submodules $e_{J} T^{r}(V)$. The chain (3.5) is a filtration of $e_{J} T^{r}(V)$ by $F G$-submodules. Also, for $\lambda \in J$, the isomorphism (3.6) is evidently an isomorphism of $F G$-modules. In view of Lemma 3.2, we have proved the following result.

Theorem 3.4 (Filtration Theorem). Let $F$ be a field of prime characteristic $p$. Let $G$ be a group and $V$ an $F G$-module. Let $e_{J}, J \in \mathcal{J}$, be primitive idempotents of the descent algebra as described above. Then the FG-module $T^{r}(V)$ 
is the direct sum of its submodules $e_{J} T^{r}(V)$. For a given $J$, let $\lambda(1), \ldots, \lambda(s)$ be the partitions belonging to $J$ in increasing lexicographic order. Then $e_{J} T^{r}(V)$ has a filtration of FG-submodules,

$$
e_{J} T^{r}(V)=U_{1} \geqslant U_{2} \geqslant \cdots \geqslant U_{s} \geqslant U_{s+1}=0,
$$

where, for $j=1, \ldots$, s, we have $U_{j} / U_{j+1} \cong L^{\lambda(j)}(V)$.

It is easily seen that if $V$ has finite dimension $n$ then each of the isomorphisms of Theorem 3.4 is an isomorphism of $S_{F}(n, r)$-modules.

Some supplementary details will be important later, so we note them in the proposition below. For each $\lambda \in \operatorname{Part}(r)$, let $\mathcal{Y}_{\lambda}$ be any subset of $W_{\lambda}$ such that the family $\left\{y+W_{\lambda+}: y \in \mathcal{Y}_{\lambda}\right\}$ consists of distinct elements forming a basis of $W_{\lambda} / W_{\lambda+}$. Thus $\bigcup \mathcal{Y}_{\lambda}$ is a basis of $T^{r}(V)$. For each $\lambda$ let $Y_{\lambda}$ be the subspace of $T^{r}(V)$ with basis $\mathcal{Y}_{\lambda}$. Let $J$ be a $p$-equivalence class of partitions of $r$. Let $\mathcal{Y}_{J}=\bigcup_{\lambda \in J} \mathcal{Y}_{\lambda}$ and let $Y_{J}$ be the subspace of $T^{r}(V)$ with basis $\mathcal{Y}_{J}$. Thus $Y_{J}=\bigoplus_{\lambda \in J} Y_{\lambda}$.

Proposition 3.5. In the notation above, the function $\phi_{J}: T^{r}(V) \rightarrow e_{J} T^{r}(V)$ given by $\phi_{J}(w)=e_{J} w$ for all $w \in T^{r}(V)$ restricts to a vector space isomorphism from $Y_{J}$ to $e_{J} T^{r}(V)$. Thus $e_{J} \mathcal{Y}_{J}$ is a basis of $e_{J} T^{r}(V)$.

Proof. By (3.7), we have

$$
\phi_{J}\left(W_{\lambda}\right)=\phi_{J}\left(W_{\lambda+}\right) \text { if } \lambda \notin J .
$$

Also, since $W_{\lambda}=Y_{\lambda}+W_{\lambda+}$, we have

$$
\phi_{J}\left(W_{\lambda}\right) \subseteq \phi_{J}\left(Y_{J}\right)+\phi_{J}\left(W_{\lambda+}\right) \text { if } \lambda \in J .
$$

It follows, by reverse induction on $\lambda$ (starting with $\lambda=(r)$ ), that $\phi_{J}\left(W_{\lambda}\right) \subseteq \phi_{J}\left(Y_{J}\right)$ for all $\lambda$. Therefore $\phi_{J}\left(T^{r}(V)\right) \subseteq \phi_{J}\left(Y_{J}\right)$ and so $\phi_{J}\left(Y_{J}\right)=\phi_{J}\left(T^{r}(V)\right)=e_{J} T^{r}(V)$. Thus the restriction $\phi_{J}: Y_{J} \rightarrow e_{J} T^{r}(V)$ is surjective. Suppose that $y$ is a non-zero element of $Y_{J}$. Then, for some $\lambda \in J$, we have $y \in W_{\lambda} \backslash W_{\lambda+}$. Since $e_{J}$ acts as the identity on $W_{\lambda} / W_{\lambda+}$, we have $\phi_{J}(y) \in W_{\lambda} \backslash W_{\lambda+}$. Thus $\phi_{J}(y) \neq 0$ and so $\phi_{J}: Y_{J} \rightarrow e_{J} T^{r}(V)$ is injective. 


\section{The Decomposition Theorem}

We begin with a major step towards the proof.

Proposition 4.1. Let $V$ be a finite-dimensional vector space over a field $F$ of prime characteristic $p$. Regard $V$ as the natural $F \mathrm{GL}(n, F)$-module, where $n=\operatorname{dim} V$. Let $q$ be a positive integer such that $q \leqslant n$ and write $q=p^{m} q^{\prime}$ where $q^{\prime}$ is not divisible by $p$. For each proper divisor $d$ of $q$, let $B_{d}$ be an $S_{F}(n, d)$ submodule of $L^{d}(V)$ such that $B_{d}$ is a direct summand of $T^{d}(V)$. Suppose that

$$
L^{p^{i} q^{\prime}}(V)=\bigoplus_{d \mid p^{i} q^{\prime}} L^{p^{i} q^{\prime} / d}\left(B_{d}\right),
$$

for $i=0, \ldots, m-1$, and suppose that the modules $L^{q / d}\left(B_{d}\right)$, where $d$ runs over the proper divisors of $q$, span their direct sum $C$ in $L^{q}(V)$. Then $L^{q}(V) / C$ is injective and projective as an $S_{F}(n, q)$-module.

Note that the notation $L^{r}\left(B_{d}\right)$ is justified by the remarks early in $\S 2$.

Proof. We first reduce to the case where $F$ is infinite. Let $E$ be an infinite extension field of $F$ and identify $E \otimes T(V)$ with $T(E \otimes V)$. Thus $E \otimes L^{r}(V)$ is identified with $L^{r}(E \otimes V)$ for all $r$. The modules $E \otimes B_{d}$ within $T(E \otimes V)$ satisfy the same hypotheses as the modules $B_{d}$ within $T(V)$. Suppose that the proposition holds over $E$. Then $E \otimes L^{q}(V) / E \otimes C$ is injective and projective as an $S_{E}(n, q)$-module. However, this module is isomorphic to $E \otimes\left(L^{q}(V) / C\right)$ and we may identify $S_{E}(n, q)$ with $E \otimes S_{F}(n, q)$. Therefore, by Lemma $2.8, L^{q}(V) / C$ is injective and projective as an $S_{F}(n, q)$-module.

Therefore we may assume that $F$ is infinite. The advantage is that $F \mathrm{GL}(n, F)$ submodules of $T^{r}(V)$ are the same as $S_{F}(n, r)$-submodules.

If $q=1$ then $C=0$ and $L^{q}(V)=V$ : thus the required conclusion follows from Lemma 2.9. Hence we may assume that $q>1$.

The remaining proof will be done in three steps. Taking careful account of the hypotheses, we first choose an ordered basis $\mathcal{A}$ of $L(V)$ which contains a basis of $C$. This yields a PBW-basis $\mathcal{F}_{q}$ of $T^{q}(V)$. We then use idempotents from various descent algebras to modify $\mathcal{F}_{q}$ to another basis $\mathcal{F}_{q}^{*}$ of $T^{q}(V)$. Certain elements of 
this modified basis will be shown to span a direct summand $U$ of $T^{q}(V)$ such that $U \cap L^{q}(V)=C$ and such that $U+L^{q}(V)$ is also a direct summand of $T^{q}(V)$. The claim about $L^{q}(V) / C$ then follows easily.

Let $\mathbf{D}$ denote the set of all proper divisors of $q$. For each $d \in \mathbf{D}$ we write $d=p^{j(d)} d^{\prime}$ where $d^{\prime}$ is not divisible by $p$. For $d \in \mathbf{D}$ and $i \in\{0, \ldots, m\}$ such that $d \mid p^{i} q^{\prime}$ (equivalently, $i \in\{j(d), \ldots, m\}$ ), choose a basis $\mathcal{B}\left(p^{i} q^{\prime} / d, d\right)$ of $L^{p^{i} q^{\prime} / d}\left(B_{d}\right)$.

For $i \in\{0, \ldots, m-1\}$ write

$$
\mathcal{A}_{p^{i} q^{\prime}}=\bigcup_{d \mid p^{i} q^{\prime}} \mathcal{B}\left(p^{i} q^{\prime} / d, d\right)
$$

Thus, by the hypotheses of the proposition, $\mathcal{A}_{p^{i} q^{\prime}}$ is a basis of $L^{p^{i} q^{\prime}}(V)$. Similarly, $\bigcup_{d \in \mathbf{D}} \mathcal{B}(q / d, d)$ is a basis of $C$. We extend this to a basis $\mathcal{A}_{q}$ of $L^{q}(V)$ : thus

$$
\mathcal{A}_{q}=\bigcup_{d \in \mathbf{D}} \mathcal{B}(q / d, d) \cup \mathcal{A}_{q}^{\prime}
$$

for some complementary set $\mathcal{A}_{q}^{\prime}$. For values of $r$ not already dealt with, let $\mathcal{A}_{r}$ be an arbitrary basis of $L^{r}(V)$, and write $\mathcal{A}=\bigcup_{r \in \mathbb{N}} \mathcal{A}_{r}$. Thus $\mathcal{A}$ is a basis of $L(V)$.

For each $d \in \mathbf{D}$ write

$$
\mathcal{B}(d)=\bigcup_{i \in\{j(d), \ldots, m\}} \mathcal{B}\left(p^{i} q^{\prime} / d, d\right)
$$

Thus

$$
\bigcup_{d \in \mathbf{D}} \mathcal{B}(d)=\left(\bigcup_{i \in\{0, \ldots, m\}} \mathcal{A}_{p^{i} q^{\prime}}\right) \backslash \mathcal{A}_{q}^{\prime} .
$$

We order $\mathcal{A}$ in any way subject to all elements of $\mathcal{B}\left(d_{1}\right)$ being smaller than all elements of $\mathcal{B}\left(d_{2}\right)$ whenever $d_{1}, d_{2} \in \mathbf{D}$ with $d_{1}<d_{2}$. As in $\S 3$, we obtain a PBW-basis $\mathcal{F}_{q}$ of $T^{q}(V)$ consisting of all elements of the form $a_{1} a_{2} \cdots a_{l}$, with $l \geqslant 0, a_{1}, \ldots, a_{l} \in \mathcal{A}, a_{1} \preccurlyeq \cdots \preccurlyeq a_{l}$ and $\operatorname{deg} a_{1} \cdots a_{l}=q$. For each $\lambda \in \operatorname{Part}(q)$, let $\mathcal{F}_{\lambda}$ and $W_{\lambda}$ be defined as in $\S 3$. Thus we have the PBW-filtration

$$
T^{q}(V)=W_{\left(1^{q}\right)} \geqslant \cdots \geqslant W_{\lambda} \geqslant W_{\lambda+} \geqslant \cdots \geqslant W_{(q)} \geqslant 0
$$

and the elements of $\mathcal{F}_{\lambda}$ taken modulo $W_{\lambda+}$ form a basis of $W_{\lambda} / W_{\lambda+}$.

If we replace each element $a_{1} a_{2} \cdots a_{l}$ of $\mathcal{F}_{\lambda}$ by any element of $W_{\lambda}$ which is equal to $a_{1} a_{2} \cdots a_{l}$ modulo $W_{\lambda+}$ we get a new set $\mathcal{F}_{\lambda}^{*}$ and if we do this for each $\lambda$ we get a new basis $\mathcal{F}_{q}^{*}=\bigcup \mathcal{F}_{\lambda}^{*}$ of $T^{q}(V)$. 
For each positive integer $r$ and each $\lambda \in \operatorname{Part}(r)$ let $\operatorname{cl}(\lambda)$ denote the $p$ equivalence class of $\lambda$ in $\operatorname{Part}(r)$. For each $r$ choose a family of primitive idempotents for the modular descent algebra $D_{r, F}$ as explained in $\S 3$. These are indexed by the $p$-equivalence classes in $\operatorname{Part}(r)$. For any divisor $k$ of $r$ we write $[r, k]$ for the partition of $r$ consisting of $r / k$ parts all equal to $k$, that is,

$$
[r, k]=\left(k^{r / k}\right)=(k, k, \ldots, k) .
$$

Furthermore, $e_{[r, k]}$ denotes the primitive idempotent of $D_{r, F}$ indexed by $\operatorname{cl}([r, k])$.

We now consider $\operatorname{cl}\left(\left[q, q^{\prime}\right]\right)$. This consists of all partitions of $q$ of the form

$$
\left(p^{m} q^{\prime}, \ldots, p^{m} q^{\prime}, \ldots, p^{i} q^{\prime}, \ldots, p^{i} q^{\prime}, \ldots, q^{\prime}, \ldots, q^{\prime}\right)
$$

where the number of occurrences of any of the $p^{i} q^{\prime}$ can be 0 . In particular, $(q) \in \operatorname{cl}\left(\left[q, q^{\prime}\right]\right)$. Note that

$$
\mathcal{F}_{(q)}=\mathcal{A}_{q}=\bigcup_{d \in \mathbf{D}} \mathcal{B}(q / d, d) \cup \mathcal{A}_{q}^{\prime}
$$

Let

$$
\mathcal{G}=\left(\bigcup_{\mu \in \mathrm{cl}\left(\left[q, q^{\prime}\right]\right)} \mathcal{F}_{\mu}\right) \backslash \mathcal{A}_{q}^{\prime}
$$

Thus, by (4.3) and the definition of $C$,

$$
\left\langle\mathcal{G} \cap L^{q}(V)\right\rangle=\bigoplus_{d \in \mathbf{D}} L^{q / d}\left(B_{d}\right)=C,
$$

where $\left\langle\mathcal{G} \cap L^{q}(V)\right\rangle$ denotes the subspace of $T(V)$ spanned by $\mathcal{G} \cap L^{q}(V)$. If $f \in \mathcal{G}$ and $f=a_{1} \cdots a_{l}$, as in the definition of $\mathcal{F}_{q}$, then each factor has degree $p^{i} q^{\prime}$ for some $i \in\{0, \ldots, m\}$ and the factors do not belong to $\mathcal{A}_{q}^{\prime}$. Thus, by (4.2),

$$
a_{1}, \ldots, a_{l} \in \bigcup_{d \in \mathbf{D}} \mathcal{B}(d) .
$$

In view of the conditions on the ordering of $\mathcal{A}$ we have $f=\prod_{d \in \mathbf{D}} f_{d}$ where the elements $d$ of $\mathbf{D}$ are taken in increasing order and where, for each $d, f_{d}$ is a product of elements of $\mathcal{B}(d)$ in increasing order.

Let $d \in \mathbf{D}$. By (4.1), each element of $\mathcal{B}(d)$ has degree divisible by $\left(q^{\prime} / d^{\prime}\right) d$. Therefore every product of elements of $\mathcal{B}(d)$ has degree $c d$ for some non-negative 
integer $c$ divisible by $q^{\prime} / d^{\prime}$. For each such $c$, write $\mathcal{G}(c, d)$ for the set of all elements of $T(V)$ of the form $a_{1} a_{2} \cdots a_{l}$ with $a_{1}, \ldots, a_{l} \in \mathcal{B}(d), a_{1} \preccurlyeq \cdots \preccurlyeq a_{l}$ and $\operatorname{deg} a_{1} \cdots a_{l}=c d$.

Let $f \in \mathcal{G}$ and write $f=\prod_{d} f_{d}$ as above. For each $d$ write $\operatorname{deg} f_{d}=c_{d} d$. Thus $c_{d}$ is divisible by $q^{\prime} / d^{\prime}$ and $f_{d} \in \mathcal{G}\left(c_{d}, d\right)$. Since $\operatorname{deg} f=q$ we have

$$
\sum_{d} c_{d} d=q
$$

Let $S$ be the set of all families $\left\{c_{d}: d \in \mathbf{D}\right\}$ of non-negative integers $c_{d}$ satisfying (4.5) such that $c_{d}$ is divisible by $q^{\prime} / d^{\prime}$ for all $d$. Note that (4.5) gives

$$
c_{d} \leqslant q / d=p^{m-j(d)} q^{\prime} / d^{\prime}
$$

For each $\sigma \in S$, where $\sigma=\left\{c_{d}: d \in \mathbf{D}\right\}$, let $\mathcal{G}(\sigma)$ be the subset of $T(V)$ defined by

$$
\mathcal{G}(\sigma)=\prod_{d} \mathcal{G}\left(c_{d}, d\right)
$$

Thus $\mathcal{G}=\bigcup_{\sigma \in S} \mathcal{G}(\sigma)$.

We next consider $\mathcal{G}\left(c_{d}, d\right)$ for fixed $d$ and $c_{d}$. Since $B_{d} \subseteq T^{d}(V)$ we may regard $T\left(B_{d}\right)$ as a subalgebra of $T(V)$, as explained early in $\S 2$. Thus $T^{c_{d}}\left(B_{d}\right) \subseteq T^{c_{d} d}(V)$. We shall modify $\mathcal{G}\left(c_{d}, d\right)$ to get a new set $\mathcal{G}^{*}\left(c_{d}, d\right)$.

Consider first the case where $c_{d} \neq 0$. We form a PBW-basis of $T^{c_{d}}\left(B_{d}\right)$ as follows. We start with bases of the Lie powers of $B_{d}$ using $\mathcal{B}\left(p^{i} q^{\prime} / d, d\right)$ as the basis of $L^{p^{i} q^{\prime} / d}\left(B_{d}\right)$ for all $i \in\{j(d), \ldots, m\}$. Hence we get a basis of $L\left(B_{d}\right)$ containing $\mathcal{B}(d)$ which we order in any way subject to agreement with the order of $\mathcal{B}(d)$ that we already have. The ordered products of degree $c_{d} d$ (as elements of $T(V)$ ) give the required PBW-basis of $T^{c_{d}}\left(B_{d}\right)$. The PBW-filtration of $T^{c_{d}}\left(B_{d}\right)$ has factors indexed by the partitions of $c_{d}$.

Consider the $p$-equivalence class $\operatorname{cl}\left(\left[c_{d}, q^{\prime} / d^{\prime}\right]\right)$. By (4.6), this class consists of all partitions of $c_{d}$ of the form

$$
\left(p^{m-j(d)} q^{\prime} / d^{\prime}, \ldots, p^{m-j(d)} q^{\prime} / d^{\prime}, \ldots, p^{i} q^{\prime} / d^{\prime}, \ldots, p^{i} q^{\prime} / d^{\prime}, \ldots, q^{\prime} / d^{\prime}, \ldots, q^{\prime} / d^{\prime}\right)
$$

The PBW-basis elements corresponding to these partitions are precisely the elements of $\mathcal{G}\left(c_{d}, d\right)$. 
Since $B_{d}$ is a module for $\mathrm{GL}(n, F)$, the action of $\operatorname{Sym}\left(c_{d}\right)$ by place permutations on $T^{c_{d}}\left(B_{d}\right)$ commutes with the action of $\mathrm{GL}(n, F)$. Thus $e_{\left[c_{d}, q^{\prime} / d^{\prime}\right]} T^{c_{d}}\left(B_{d}\right)$ is a direct summand of $T^{c_{d}}\left(B_{d}\right)$ as an $F \mathrm{GL}(n, F)$-module. By hypothesis, $B_{d}$ is a direct summand of $T^{d}(V)$, and so $e_{\left[c_{d}, q^{\prime} / d^{\prime}\right]} T^{c_{d}}\left(B_{d}\right)$ is a direct summand of $T^{c_{d} d}(V)$.

For each $f_{d} \in \mathcal{G}\left(c_{d}, d\right)$ define $f_{d}^{*}=e_{\left[c_{d}, q^{\prime} / d^{\prime}\right]} f_{d}$ and write

$$
\mathcal{G}^{*}\left(c_{d}, d\right)=\left\{f_{d}^{*}: f_{d} \in \mathcal{G}\left(c_{d}, d\right)\right\} .
$$

By Proposition 3.5 (applied to the module $B_{d}$ rather than $V$ ),

$$
\left\langle\mathcal{G}^{*}\left(c_{d}, d\right)\right\rangle=e_{\left[c_{d}, q^{\prime} / d^{\prime}\right]} T^{c_{d}}\left(B_{d}\right) .
$$

Thus $\left\langle\mathcal{G}^{*}\left(c_{d}, d\right)\right\rangle$ is an $F \mathrm{GL}(n, F)$-module which is a direct summand of $T^{c_{d} d}(V)$.

If $c_{d}=0$ we have $\mathcal{G}\left(c_{d}, d\right)=\{1\}$. We define $1^{*}=1$. Thus we take $\mathcal{G}^{*}\left(c_{d}, d\right)=\{1\}$ and $\left\langle\mathcal{G}^{*}\left(c_{d}, d\right)\right\rangle=F .1=T^{0}(V)$.

For $f \in \mathcal{G}(\sigma)$, where $\sigma=\left\{c_{d}: d \in \mathbf{D}\right\}$ and $f=\prod_{d} f_{d}$ as above, we define $f^{*}=\prod_{d} f_{d}^{*}$. For each $d,(3.4)$ gives that $f_{d}^{*}$ is equal to $f_{d}$ modulo terms below $f_{d}$ in the PBW-filtration of $T^{c_{d}}\left(B_{d}\right)$. It follows that $f^{*}$ is equal to $f$ modulo terms below $f$ in the PBW-filtration of $T^{q}(V)$. Hence $f$ may be replaced by $f^{*}$ in the basis $\mathcal{F}_{q}$ of $T^{q}(V)$.

This procedure applied to all $\sigma$ gives $f^{*}$ for all $f \in \mathcal{G}=\bigcup_{\sigma} \mathcal{G}(\sigma)$. For $f \in \mathcal{F}_{q} \backslash \mathcal{G}$ we define $f^{*}=f$, and write $\mathcal{F}_{q}^{*}=\left\{f^{*}: f \in \mathcal{F}_{q}\right\}$. Thus $\mathcal{F}_{q}^{*}$ is a basis of $T^{q}(V)$. For each $\lambda \in \operatorname{Part}(q)$ we also write $\mathcal{F}_{\lambda}^{*}=\left\{f^{*}: f \in \mathcal{F}_{\lambda}\right\}$. Note that if $f \in \mathcal{F}_{(q)}$ then $f$ belongs to the bottom term of the PBW-filtration of $T^{q}(V)$ and so $f^{*}=f$.

Recall that $\mathcal{G}^{*}\left(c_{d}, d\right)$ is given by (4.8). Similarly, write

$$
\mathcal{G}^{*}(\sigma)=\left\{f^{*}: f \in \mathcal{G}(\sigma)\right\},
$$

for all $\sigma \in S$, and

$$
\mathcal{G}^{*}=\left\{f^{*}: f \in \mathcal{G}\right\}=\bigcup_{\sigma} \mathcal{G}^{*}(\sigma) .
$$

By (4.7), if $\sigma=\left\{c_{d}: d \in \mathbf{D}\right\}$, we have

$$
\mathcal{G}^{*}(\sigma)=\prod_{d} \mathcal{G}^{*}\left(c_{d}, d\right) .
$$

Thus

$$
\left\langle\mathcal{G}^{*}(\sigma)\right\rangle=\prod_{d}\left\langle\mathcal{G}^{*}\left(c_{d}, d\right)\right\rangle,
$$


where the right-hand side denotes the span of the product of the sets $\left\langle\mathcal{G}^{*}\left(c_{d}, d\right)\right\rangle$ within $T(V)$. Since $\mathcal{G}^{*}(\sigma) \subseteq \mathcal{F}_{q}^{*}$, it follows that $\mathcal{G}^{*}(\sigma)$ is a basis of $\left\langle\mathcal{G}^{*}(\sigma)\right\rangle$. For a similar reason the spaces $\left\langle\mathcal{G}^{*}(\sigma)\right\rangle, \sigma \in S$, span their direct sum in $T^{q}(V)$. Let $U$ be this direct sum,

$$
U=\bigoplus_{\sigma \in S}\left\langle\mathcal{G}^{*}(\sigma)\right\rangle .
$$

Thus, by (4.9), $U=\left\langle\mathcal{G}^{*}\right\rangle$. Also, since $\mathcal{F}_{(q)}^{*}=\mathcal{F}_{(q)}$,

$$
L^{q}(V)=\left\langle\left(\mathcal{G} \cap L^{q}(V)\right) \cup \mathcal{A}_{q}^{\prime}\right\rangle=\left\langle\left(\mathcal{G}^{*} \cap L^{q}(V)\right) \cup \mathcal{A}_{q}^{\prime}\right\rangle
$$

Hence $U+L^{q}(V)=\left\langle\mathcal{G}^{*} \cup \mathcal{A}_{q}^{\prime}\right\rangle$ and $U \cap L^{q}(V)=\left\langle\mathcal{G} \cap L^{q}(V)\right\rangle$. Thus, by (4.4),

$$
U \cap L^{q}(V)=C
$$

Since each $\left\langle\mathcal{G}^{*}\left(c_{d}, d\right)\right\rangle$ is an $F \mathrm{GL}(n, F)$-submodule of $T(V)$, so is $\left\langle\mathcal{G}^{*}(\sigma)\right\rangle$, by (4.10). Indeed, since $\left\langle\mathcal{G}^{*}\left(c_{d}, d\right)\right\rangle$ is a direct summand of $T^{c_{d} d}(V)$ for all $d$, it follows that $\left\langle\mathcal{G}^{*}(\sigma)\right\rangle$ is a direct summand of $T^{q}(V)$. Thus, by Lemma 2.9, each $\left\langle\mathcal{G}^{*}(\sigma)\right\rangle$ is injective as an $S_{F}(n, q)$-module. Thus, by (4.11), $U$ is injective.

Let $\phi: T^{q}(V) \rightarrow e_{\left[q, q^{\prime}\right]} T^{q}(V)$ be the mapping given by $\phi(w)=e_{\left[q, q^{\prime}\right]} w$ for all $w \in T^{q}(V)$. Thus (see $\left.\S 3\right) \phi$ is a homomorphism of $F \mathrm{GL}(n, F)$-modules; hence a homomorphism of $S_{F}(n, q)$-modules. As shown above, $U+L^{q}(V)$ is the subspace of $T^{q}(V)$ spanned by $\mathcal{G}^{*} \cup \mathcal{A}_{q}^{\prime}$. However,

$$
\mathcal{G}^{*} \cup \mathcal{A}_{q}^{\prime}=\bigcup_{\mu \in \operatorname{cl}\left(\left[q, q^{\prime}\right]\right)} \mathcal{F}_{\mu}^{*}
$$

Hence, by Proposition 3.5, the restriction of $\phi$ is a vector space isomorphism from $U+L^{q}(V)$ to $e_{\left[q, q^{\prime}\right]} T^{q}(V)$. Thus it is an $S_{F}(n, q)$-module isomorphism. Therefore, by Lemma 2.9, $U+L^{q}(V)$ is injective and projective. (Hence it is a direct summand of $T^{q}(V)$.) However, by (4.12),

$$
L^{q}(V) / C \cong\left(U+L^{q}(V)\right) / U
$$

Since $U$ is injective, it follows that $L^{q}(V) / C$ is injective and projective. This completes the proof of Proposition 4.1.

We can now state our key result. We refer to $\S 2$ for the notation $V^{(n)}$ and definitions concerning uniform families of modules. 
THEOREM 4.2. Let $F$ be a field and $k$ a positive integer not divisible by the characteristic of $F$. For each positive integer $s$ there exists a uniform family $\left\{B_{s k}^{(n)}: n \in \mathbb{N}\right\}$ of modules of degree sk such that, for all $n, B_{s k}^{(n)}$ is a submodule of $L^{s k}\left(V^{(n)}\right)$ and a direct summand of $T^{s k}\left(V^{(n)}\right)$ and

$$
L^{k}\left(V^{(n)}\right) \oplus L^{2 k}\left(V^{(n)}\right) \oplus L^{3 k}\left(V^{(n)}\right) \oplus \cdots=L\left(B_{k}^{(n)}\right) \oplus L\left(B_{2 k}^{(n)}\right) \oplus L\left(B_{3 k}^{(n)}\right) \oplus \cdots
$$

Before proving Theorem 4.2 we show that it implies our main results for an arbitrary group $G$.

THEOREM 4.3. Let $F$ be a field and $k$ a positive integer not divisible by the characteristic of $F$. Let $G$ be a group and $V$ a finite-dimensional FG-module. For each positive integer $s$ there is a submodule $B_{s k}$ of $L^{s k}(V)$ such that $B_{s k}$ is a direct summand of $T^{s k}(V)$ and

$$
L^{s k}(V)=\bigoplus_{c \mid s} L^{s / c}\left(B_{c k}\right)
$$

Proof. Let $n=\operatorname{dim} V$. By choice of a basis of $V$, the representation of $G$ on $V$ gives a homomorphism $\theta: G \rightarrow \mathrm{GL}(n, F)$. We can regard $V$ as the natural module for $\operatorname{GL}(n, F)$. For each positive integer $s$ define $B_{s k}=B_{s k}^{(n)}$ where $B_{s k}^{(n)}$ is as given by Theorem 4.2. The action of $G$ on $T(V)$ is the composite of $\theta$ and the action of $\mathrm{GL}(n, F)$ on $T(V)$. Hence, for each $s, B_{s k}$ is an $F G$-submodule of $L^{s k}(V)$ and a direct summand of $T^{s k}(V)$, and $\bigoplus_{s} L^{s k}(V)=\bigoplus_{s} L\left(B_{s k}\right)$. By comparing terms of degree $s k$ we obtain $L^{s k}(V)=\bigoplus_{c \mid s} L^{s / c}\left(B_{c k}\right)$.

Theorem 4.4 (Decomposition Theorem). Let $F$ be a field of prime characteristic $p$. Let $G$ be a group and $V$ a finite-dimensional FG-module. For each positive integer $r$ there is a submodule $B_{r}$ of $L^{r}(V)$ such that $B_{r}$ is a direct summand of $T^{r}(V)$ and, for $k$ not divisible by $p$ and $m \geqslant 0$,

$$
L^{p^{m} k}(V)=L^{p^{m}}\left(B_{k}\right) \oplus L^{p^{m-1}}\left(B_{p k}\right) \oplus \cdots \oplus L^{1}\left(B_{p^{m} k}\right) .
$$


Proof. For each positive integer $k$ not divisible by $p$, take $B_{k}, B_{p k}, B_{p^{2} k}, \ldots$ as given by Theorem 4.3. The result follows.

Proof of Theorem 4.2. We shall consider various families $\left\{U^{(n)}: n \in \mathbb{N}\right\}$ where, for each $n, U^{(n)}$ is a subspace of $T\left(V^{(n)}\right)$. When $n$ is regarded as fixed but arbitrary we generally simplify the notation by writing $U$ instead of $U^{(n)}$. In particular, we often write $V$ for $V^{(n)}$.

Let $Q=Q^{(n)}=L^{k}(V) \oplus L^{2 k}(V) \oplus L^{3 k}(V) \oplus \cdots$. Clearly $Q$ is a subalgebra of $L(V)$ and $Q=\bigoplus_{r \geqslant 1} Q_{r}$ where $Q_{r}=Q \cap L^{r}(V)$ for all $r$. Of course, $Q_{r}=0$ unless $r$ is divisible by $k$ and $Q_{s k}=L^{s k}(V)$ for all $s$. For each $r \geqslant 1$, let $Q(r)$ denote the subalgebra of $Q$ generated by $Q_{1} \oplus \cdots \oplus Q_{r}$. Also, write $Q(0)=0$.

For each $r \geqslant 1$, let $W_{r}$ (or $W_{r}^{(n)}$ ) be any subspace of $Q_{r}$ with the property that $Q_{r}=\left(Q(r-1) \cap Q_{r}\right) \oplus W_{r}$. The choice of these subspaces is arbitrary at present: later we make a more careful choice. By Lemma 2.1, we have $Q=L\left(W_{1} \oplus W_{2} \oplus \cdots\right)$. Clearly $W_{r}=0$ unless $r=s k$ for some $s$. Hence $Q=L\left(W_{k} \oplus W_{2 k} \oplus W_{3 k} \oplus \cdots\right)$. Also, $W_{k}=L^{k}(V)$.

For each positive integer $s$ we construct a subspace $B_{s k}$ of $L^{s k}(V)$ such that $Q=L\left(B_{k}\right) \oplus L\left(B_{2 k}\right) \oplus \cdots$. For each $s, B_{s k}$ depends only on $W_{k}, W_{2 k}, \ldots, W_{s k}$.

Step 1. Define $B_{k}=W_{k}$ and $C_{k}=W_{2 k} \oplus W_{3 k} \oplus \cdots$. By Lemma 2.2,

$$
Q=L\left(B_{k} \oplus C_{k}\right)=L\left(B_{k}\right) \oplus L\left(C_{k} \curlywedge B_{k}\right)
$$

Also, by Corollary $2.3, C_{k} \prec B_{k}$ is the direct sum of the subspaces in $\mathcal{E}_{1}$, where

$$
\mathcal{E}_{1}=\left\{[W_{i k}, \underbrace{B_{k}, \ldots, B_{k}}_{r}]: i>1, r \geqslant 0\right\}
$$

Step 2. Let $B_{2 k}$ be the sum of all elements of $\mathcal{E}_{1}$ of degree $2 k$ (so that, in fact, $\left.B_{2 k}=W_{2 k}\right)$ and let $C_{2 k}$ be the sum of all elements of $\mathcal{E}_{1}$ of degree greater than $2 k$. By Lemma 2.2,

$$
L\left(C_{k} \prec B_{k}\right)=L\left(B_{2 k}\right) \oplus L\left(C_{2 k} \prec B_{2 k}\right) .
$$

Also, by Corollary 2.3, $C_{2 k} \prec B_{2 k}$ is the direct sum of the subspaces in $\mathcal{E}_{2}$, where

$$
\mathcal{E}_{2}=\left\{[E, \underbrace{B_{2 k}, \ldots, B_{2 k}}_{r}]: E \in \mathcal{E}_{1}, \operatorname{deg} E>2 k, r \geqslant 0\right\} .
$$


We continue in this way. In Step $s$ (where $s>1$ ) we define $B_{s k}$ to be the sum of all elements of $\mathcal{E}_{s-1}$ of degree $s k$ and $C_{s k}$ to be the sum of all elements of $\mathcal{E}_{s-1}$ of degree greater than sk. By Lemma 2.2 and Corollary 2.3,

$$
L\left(C_{(s-1) k} \prec B_{(s-1) k}\right)=L\left(B_{s k}\right) \oplus L\left(C_{s k} \prec B_{s k}\right)
$$

and $C_{s k} \curlyvee B_{s k}$ is the direct sum of the subspaces in $\mathcal{E}_{s}$, where

$$
\mathcal{E}_{s}=\left\{[E, \underbrace{B_{s k}, \ldots, B_{s k}}_{r}]: E \in \mathcal{E}_{s-1}, \operatorname{deg} E>s k, r \geqslant 0\right\}
$$

In particular, every subspace in $\mathcal{E}_{s}$ has the form

$$
\left[W_{i k}, B_{s(1) k}, \ldots, B_{s(r) k}\right]
$$

where $i>1, r \geqslant 0$ and $1 \leqslant s(1) \leqslant \cdots \leqslant s(r) \leqslant s$.

By (4.13), (4.14) and considerations of degree, we obtain

$$
Q=L\left(B_{k}\right) \oplus L\left(B_{2 k}\right) \oplus L\left(B_{3 k}\right) \oplus \cdots
$$

This will give the statement of the theorem if we can show that the families $\left\{W_{s k}^{(n)}\right\}$ may be chosen so that the resulting families $\left\{B_{s k}^{(n)}\right\}$ are uniform families of modules with $B_{s k}^{(n)}$ a direct summand of $T^{s k}\left(V^{(n)}\right)$ for all $n$ and $s$.

Comparing terms of degree $s k$ in $(4.15)$ we have, for all $s \geqslant 1$,

$$
L^{s k}(V)=Q_{s k}=\bigoplus_{c \mid s} L^{s / c}\left(B_{c k}\right) .
$$

As at the end of $\S 2$, let $\mathcal{T}_{r}^{(n)}$ (or $\mathcal{T}_{r}$ when $n$ is understood) denote the class of all finite-dimensional $S_{F}(n, r)$-modules that are direct sums of modules each of which is isomorphic to a direct summand of $T^{r}\left(V^{(n)}\right)$. By Lemma 2.10 it suffices to obtain uniform families $\left\{B_{s k}^{(n)}\right\}$ such that $B_{s k}^{(s k)} \in \mathcal{T}_{s k}^{(s k)}$ for all $s$.

We start with arbitrary families $\left\{W_{s k}^{(n)}\right\}$ and then refine the choice of $\left\{W_{k}^{(n)}\right\}$, $\left\{W_{2 k}^{(n)}\right\}, \ldots$, successively, so that the resulting families $\left\{W_{s k}^{(n)}\right\}$ and $\left\{B_{s k}^{(n)}\right\}$ are uniform families of modules satisfying $W_{s k}^{(n)}, B_{s k}^{(n)} \in \mathcal{T}_{s k}^{(n)}$ for all $n$ and $s$.

There is no choice possible for $W_{k}$ : we have $W_{k}=L^{k}(V)$. Step 1 of the construction described above gives $B_{k}=W_{k}$. Thus the families $\left\{W_{k}^{(n)}\right\}$ and $\left\{B_{k}^{(n)}\right\}$ are uniform families of modules of degree $k$. By a well-known result (see, for 
example, $[\mathbf{1 3}, \S 3.1]), L^{k}(V)$ is a direct summand of $T^{k}(V)$, because $k$ is not divisible by the characteristic of $F$. Thus $W_{k} \in \mathcal{T}_{k}$ and $B_{k} \in \mathcal{T}_{k}$.

Suppose that $s \geqslant 2$ and that we have chosen the families $\left\{W_{i k}^{(n)}\right\}$ for all $i<s$ so that $\left\{W_{i k}^{(n)}\right\}$ and $\left\{B_{i k}^{(n)}\right\}$ are uniform families of modules belonging to $\mathcal{T}_{i k}$. We now consider Step $s$.

By definition, $B_{s k}$ is the sum of all elements of $\mathcal{E}_{s-1}$ of degree $s k$. One such term is $W_{s k}$. The other terms have the form $\left[W_{i k}, B_{s(1) k}, \ldots, B_{s(r) k}\right]$ with $r>0$. Let $U_{s k}$ denote the sum of these latter terms. Thus $B_{s k}=U_{s k} \oplus W_{s k}$. Consider a summand $\left[W_{i k}, B_{s(1) k}, \ldots, B_{s(r) k}\right]$ of $U_{s k}$. Since $r>0$, we have $i<s$ and $s(1), \ldots, s(r)<s$. Hence $\left\{W_{i k}^{(n)}\right\},\left\{B_{s(1) k}^{(n)}\right\}, \ldots,\left\{B_{s(r) k}^{(n)}\right\}$ are uniform families of modules belonging to $\mathcal{T}_{i k}, \mathcal{T}_{s(1) k}, \ldots, \mathcal{T}_{s(r) k}$, respectively.

Repeated use of Corollary 2.3 gives a vector space isomorphism

$$
\left[W_{i k}, B_{s(1) k}, \ldots, B_{s(r) k}\right] \cong W_{i k} \otimes B_{s(1) k} \otimes \cdots \otimes B_{s(r) k}
$$

It is easy to check, by Lemma 2.4 , that these spaces are $S_{F}(n, s k)$-modules and the isomorphism is a module isomorphism. It follows that $\left[W_{i k}, B_{s(1) k}, \ldots, B_{s(r) k}\right] \in \mathcal{T}_{s k}$. Also, by Lemma 2.7 , the family $\left\{\left[W_{i k}^{(n)}, B_{s(1) k}^{(n)}, \ldots, B_{s(r) k}^{(n)}\right]\right\}$ is uniform. It follows that $\left\{U_{s k}^{(n)}\right\}$ is a uniform family of modules belonging to $\mathcal{T}_{s k}$.

By (4.16),

$$
L^{s k}(V)=Q_{s k}=\bigoplus_{c \mid s, c<s} L^{s / c}\left(B_{c k}\right) \oplus U_{s k} \oplus W_{s k}
$$

Note that

$$
\bigoplus_{c \mid s, c<s} L^{s / c}\left(B_{c k}\right) \oplus U_{s k} \subseteq Q(s k-1) \cap Q_{s k}
$$

and, by the defining property of $W_{s k}$,

$$
Q_{s k}=\left(Q(s k-1) \cap Q_{s k}\right) \oplus W_{s k} .
$$

Thus, by (4.17),

$$
\bigoplus_{c \mid s, c<s} L^{s / c}\left(B_{c k}\right) \oplus U_{s k}=Q(s k-1) \cap Q_{s k}
$$

We next prove that the module

$$
L^{s k}\left(V^{(s k)}\right) / \bigoplus_{c \mid s, c<s} L^{s / c}\left(B_{c k}^{(s k)}\right)
$$


is injective and projective as an $S_{F}(s k, s k)$-module. This is clear if $F$ has characteristic 0 because in that case $S_{F}(s k, s k)$ is semisimple, by $[\mathbf{1 6},(2.6 \mathrm{e})]$. Thus we may suppose that $F$ has prime characteristic $p$. By hypothesis, $p \nmid k$. For each positive integer $r$ not divisible by $k$, define $B_{r}^{(s k)}=0$. In Proposition 4.1, take $n=q=s k, V=V^{(s k)}$ and $B_{d}=B_{d}^{(s k)}$ for every proper divisor $d$ of $q$. The hypotheses of Proposition 4.1 follow from (4.16). Thus the module (4.20) is injective and projective, as required.

By (4.17), this module has a submodule

$$
\left(\bigoplus_{c \mid s, c<s} L^{s / c}\left(B_{c k}^{(s k)}\right) \oplus U_{s k}^{(s k)}\right) / \bigoplus_{c \mid s, c<s} L^{s / c}\left(B_{c k}^{(s k)}\right)
$$

isomorphic to $U_{s k}^{(s k)}$. By Lemma 2.9, $U_{s k}^{(s k)}$ is injective. It follows that

$$
L^{s k}\left(V^{(s k)}\right) /\left(\bigoplus_{c \mid s, c<s} L^{s / c}\left(B_{c k}^{(s k)}\right) \oplus U_{s k}^{(s k)}\right)
$$

is injective and projective. Hence there exists $Z_{s k}^{(s k)} \in \mathcal{T}_{s k}^{(s k)}$ such that

$$
L^{s k}\left(V^{(s k)}\right)=\bigoplus_{c \mid s, c<s} L^{s / c}\left(B_{c k}^{(s k)}\right) \oplus U_{s k}^{(s k)} \oplus Z_{s k}^{(s k)} .
$$

By Lemma 2.5, $Z_{s k}^{(s k)}$ extends to a uniform family $\left\{Z_{s k}^{(n)}\right\}$ of modules of degree $s k$. Also, by Lemma 2.10, $Z_{s k}^{(n)} \in \mathcal{T}_{s k}^{(n)}$ for all $n$. Furthermore, by Lemma 2.7, $\left\{L^{s / c}\left(B_{c k}^{(n)}\right)\right\}$ is uniform, for all proper divisors $c$ of $s$, and we have seen that $\left\{U_{s k}^{(n)}\right\}$ is uniform. Hence, by Lemma 2.6, we have (for arbitrary $n$ )

$$
L^{s k}(V)=\bigoplus_{c \mid s, c<s} L^{s / c}\left(B_{c k}\right) \oplus U_{s k} \oplus Z_{s k}
$$

Therefore, in view of (4.19),

$$
Q_{s k}=L^{s k}(V)=\left(Q(s k-1) \cap Q_{s k}\right) \oplus Z_{s k}
$$

However, (4.18) is the defining property of $W_{s k}$. Thus we may replace our arbitrary choice of $W_{s k}$ by taking $W_{s k}=Z_{s k}$. Recall that $B_{s k}=U_{s k} \oplus W_{s k}$. Thus $\left\{W_{s k}^{(n)}\right\}$ and $\left\{B_{s k}^{(n)}\right\}$ are uniform families of modules belonging to $\mathcal{T}_{s k}$.

This completes the proof of Theorem 4.2.

Acknowledgements. The authors are grateful to Dr K. Erdmann and Dr R. Stöhr for their useful comments while this work was in progress. 


\section{References}

1. D. Blessenohl and H. Laue, 'The module structure of Solomon's descent algebra', J. Austral. Math. Soc. 72 (2002) 317-333.

2. N. Bourbaki, Lie groups and Lie algebras, Part I: Chapters 1-3 (Hermann, Paris, 1975).

3. A. J. BRANDT, 'The free Lie ring and Lie representations of the full linear group', Trans. Amer. Math. Soc. 56 (1944) 528-536.

4. R. M. Bryant, 'Free Lie algebras and Adams operations', J. London Math. Soc. (2) 68 (2003) 355-370.

5. R. M. BRYAnt, 'Modular Lie representations of groups of prime order', Math. Z. 246 (2004) 603-617.

6. R. M. Bryant, 'Modular Lie representations of finite groups', J. Austral. Math. Soc. 77 (2004) 401-423.

7. R. M. Bryant, L. G. Kovács and R. StöHR, 'Lie powers of modules for groups of prime order', Proc. London Math. Soc. (3) 84 (2002) 343-374.

8. R. M. Bryant and I. C. Michos, 'Lie powers of free modules for certain groups of prime power order', J. Austral. Math. Soc. 71 (2001) 149-158.

9. R. M. Bryant and M. SCHOCKER, 'Lie powers and Lie resolvents', in preparation.

10. R. M. Bryant and R. StöHR, 'Lie powers in prime degree', Quart. J. Math., to appear.

11. H. Cartan and S. Eilenberg, Homological algebra (Princeton University Press, Princeton, 1956).

12. S. Donkin, 'On tilting modules for algebraic groups', Math. Z. 212 (1993) $39-60$.

13. S. Donkin and K. ERDMAnN, 'Tilting modules, symmetric functions, and the module structure of the free Lie algebra', J. Algebra 203 (1998) 69-90.

14. K. Erdmann and M. Schocker, 'Modular Lie powers and the Solomon descent algebra', preprint.

15. A. M. Garsia and C. Reutenauer, 'A decomposition of Solomon's descent algebra', Adv. Math. 77 (1989) 189-262. 
16. J. A. Green, Polynomial representations of $\mathrm{GL}_{n}$, Lecture Notes in Mathematics 830 (Springer, Berlin, 1980).

17. B. Hartley and R. StöHr, 'Homology of higher relation modules and torsion in free central extensions of groups', Proc. London Math. Soc. (3) 62 (1991) 325-352.

18. N. JaCobson, Lie algebras (Interscience, New York, 1962).

19. A. A. Klyachko, 'Lie elements in the tensor algebra', Siberian Math. J. 15 (1974) 914-921 (1975).

20. W. KRASKIEWICZ and J. WEYMAN, 'Algebra of coinvariants and the action of a Coxeter element', Bayreuth. Math. Schr. 63 (2001) 265-284.

21. C. Reutenauer, Free Lie algebras (Clarendon Press, Oxford, 1993).

R. M. Bryant

School of Mathematics

University of Manchester

PO Box 88

Manchester M60 1QD

roger.bryant@manchester.ac.uk
M. Schocker

Department of Mathematics

University of Wales Swansea

Singleton Park

Swansea SA2 8PP

m.schocker@swansea.ac.uk 